\title{
Instability and transition to turbulence in a free shear layer affected by a parallel magnetic field
}

\author{
A. VOROBEV AND O. ZIKANOV† \\ Department of Mechanical Engineering, University of Michigan - Dearborn, 4901 Evergreen Road, \\ Dearborn, MI 48128-1491, USA
}

(Received 4 May 2006 and in revised form 25 September 2006)

Instability and transition to turbulence in a temporally evolving free shear layer of an electrically conducting fluid affected by an imposed parallel magnetic field is investigated numerically. The case of low magnetic Reynolds number is considered. It has long been known that the neutral disturbances of the linear problem are three-dimensional at sufficiently strong magnetic fields. We analyse the details of this instability solving the generalized Orr-Sommerfeld equation to determine the wavenumbers, growth rates and spatial shapes of the eigenmodes. The threedimensional perturbations are identified as oblique waves and their properties are described. In particular, we find that at high hydrodynamic Reynolds number, the effect of the strength of the magnetic field on the fastest growing perturbations is limited to an increase of their oblique angle. The dimensions and spatial shape of the waves remain unchanged. The transition to turbulence triggered by the growing oblique waves is investigated in direct numerical simulations. It is shown that initial perturbations in the form of superposition of two symmetric waves are particularly effective in inducing three-dimensionality and turbulence in the flow.

\section{Introduction}

We consider the instability and subsequent transition to turbulence in a free shear layer of an incompressible viscous electrically conducting fluid with the initial velocity profile

$$
\boldsymbol{U}=(U(z), 0,0), \quad U(z) \rightarrow \pm U_{0} \quad \text { at } \quad z \rightarrow \pm \infty .
$$

A uniform time-independent magnetic field $\boldsymbol{B}=(B, 0,0)$ in the streamwise direction is imposed in the entire flow domain. We assume that the magnetic Reynolds number is small

$$
R e_{m} \equiv \frac{U_{0} L}{\lambda} \ll 1 .
$$

Here, $L$ is the length scale, which we take to be the initial vorticity thickness of the layer, and $\lambda=\left(\sigma \mu_{0}\right)^{-1}$ is the magnetic diffusivity, with $\sigma$ being the electrical conductivity of the fluid and $\mu_{0}$ the magnetic permeability of a vacuum. The condition (1.2) is typical for laboratory and industrial flows of liquid metals, molten oxides, and other electrically conducting materials. It allows us to apply the low- $R e_{m}$ approximation (Davidson 2001), according to which the perturbations of the magnetic field induced by the fluid motion adjust instantaneously to the variations of the flow 
and can be neglected in comparison with the imposed field $\boldsymbol{B}$ in the expression for the Lorentz force.

In the approximation, the non-dimensional equations of motion are

$$
\frac{\partial \boldsymbol{v}}{\partial t}+(\boldsymbol{v} \cdot \nabla) \boldsymbol{v}=-\nabla p+\frac{1}{R e} \nabla^{2} \boldsymbol{v}+N(\boldsymbol{j} \times \boldsymbol{B}), \quad \nabla \cdot \boldsymbol{v}=0,
$$

where the electric current $\boldsymbol{j}$ is calculated according to

$$
\boldsymbol{j}=-\nabla \phi+\boldsymbol{v} \times \boldsymbol{B}
$$

with the electric potential $\phi$ determined as a solution of the Poisson equation

$$
\nabla^{2} \phi=\boldsymbol{B} \cdot(\nabla \times \boldsymbol{v})
$$

with proper boundary conditions. The non-dimensional parameters are the Reynolds number and the magnetic interaction parameter

$$
R e \equiv \frac{U_{0} L}{v}, \quad N \equiv \frac{\sigma B^{2} L}{\rho U_{0}} .
$$

The value of $N$ gives an estimate to the ratio between the Lorentz and inertia forces, thus evaluating the potential of the magnetic field to suppress and transform the perturbations.

No electric currents and Lorentz forces are generated in the unperturbed basic flow (1.1). This can be verified easily by taking the curl of the Ohm's law (1.4), which leads to

$$
\nabla \times j=(B \cdot \nabla) v .
$$

For any velocity field, which is uniform in the direction of the magnetic field, the right-hand side of (1.7) is zero and the only solution in the absence of externally applied electric currents is $\boldsymbol{j}=0$.

The basic flow (1.1) can be viewed as a simplified model of shear layers coplanar to a strong imposed magnetic field, which can appear in low- $R e_{m}$ magnetohydrodynamics (MHD) in such common situations as thermal convection, jets, wakes behind bodies, or at sharp non-uniformities of the field or electromagnetic boundary conditions (see, e.g. Moreau 1990; Davidson 2001; Müller \& Bühler 2001). It can also be considered as a representative of parallel vortex sheets developing in strongly anisotropic MHD turbulence (Zikanov \& Thess 1998). The importance and seeming simplicity of the flow caused attention to it in the past. We will briefly review the history of the subject before proceeding to our contribution.

Stability of a shear velocity profile in the presence of a parallel magnetic field was first analysed by Michael (1953), Stuart (1954) and Drazin (1960). Linear instability to normal modes

$$
\boldsymbol{v}^{\prime}(x, y, z, t)=\boldsymbol{v}(z) \exp \left[\mathrm{i}\left(k_{x} x+k_{y} y-\lambda t\right)\right]
$$

was considered. Here, $k_{x}$ and $k_{y}$ are the real wavenumbers in the $x$-and $y$-directions, and $\lambda=\omega+\mathrm{i} \beta$ is the complex phase velocity. The basic flow is unstable if at least one solution (1.8) has $\beta>0$. Analysing the solutions of the generalized OrrSommerfeld equation for several basic velocity profiles, it was found that the magnetic field stabilizes the basic flow because of the additional suppression of the growing perturbation by the Joule dissipation. The analysis was, however, based on the erroneous assumption that the Squire transformation could be applied to the MHD stability problem with the same consequences as in the classical non-magnetic case. Only two-dimensional disturbances with $k_{y}=0$ were considered. 
The correct view was taken by Hunt (1966), who showed that the three-dimensional perturbations could cause instability at lower Reynolds numbers than the twodimensional ones if the magnetic field was sufficiently strong. The reasoning was simple. If we consider an arbitrary mode (1.8) with $k_{y} \neq 0$, the non-dimensional generalized Orr-Sommerfeld problem becomes

$$
\left(k_{x} U-\lambda\right)\left(v_{z}^{\prime \prime}-k^{2} v_{z}\right)-k_{x} v_{z} U^{\prime \prime}+\mathrm{i} k_{x}^{2} N v_{z}=-\frac{\mathrm{i}}{R e}\left(v_{z}^{\mathrm{iv}}-2 k^{2} v_{z}^{\prime \prime}+k^{4} v_{z}\right), \quad \lim _{z \rightarrow \pm \infty} v_{z}=0
$$

Here, $v_{z}$ is the $z$-component of the perturbation velocity, $k=\left(k_{x}^{2}+k_{y}^{2}\right)^{1 / 2}$ is the wavenumber, and primes stand for the derivatives in the $z$-direction. We can define the oblique angle $\theta=\cos ^{-1}\left(k_{x} / k\right)$ as the angle between the direction of the wave propagation and the basic flow.

Following Squire (1933), we can rewrite (1.9) as

$$
(U-\widehat{\lambda})\left(v_{z}^{\prime \prime}-k^{2} v_{z}\right)-v_{z} U^{\prime \prime}+\mathrm{i} k \widehat{N} v_{z}=-\frac{\mathrm{i}}{k \widehat{R e}}\left(v_{z}^{\mathrm{iv}}-2 k^{2} v_{z}^{\prime \prime}+k^{4} v_{z}\right), \quad \lim _{z \rightarrow \pm \infty} v_{z}=0,
$$

where the new non-dimensional parameters are $\widehat{R e}=\left(k_{x} / k\right) \operatorname{Re}$ and $\widehat{N}=\left(k_{x} / k\right) N$, and $\widehat{\lambda}=\lambda / k_{x}$. The solution of the problem can be expressed as a relation between the parameters, the wavenumber, and the eigenvalue $\hat{\lambda}$

$$
F(\widehat{\lambda}, k, \widehat{R e}, \widehat{N})=0,
$$

which is the same for any angle $\theta$ and, thus, can be determined assuming twodimensional waves with $\theta=0$. Let us now consider the critical Reynolds number $R e_{c}$ defined as the minimum Re occurring over all $k$ and $\omega$, at which a neutral mode with $\beta=0$ is observed. From (1.11), we find

$$
F_{c}\left(\widehat{R e_{c}}, \widehat{N}\right)=0 \quad \text { or } \quad \widehat{\operatorname{Re}_{c}}=G(\widehat{N}) .
$$

In the non-magnetic case with $N=0$, this results in the Squire theorem that the two-dimensional perturbations are always first to become unstable since the smallest critical Reynolds number $R e_{c}=\left(k / k_{x}\right) \widehat{R e}_{c}=\widehat{R e}_{c} / \cos \theta$ is always for the perturbations with $\theta=0$. If, on the other hand, $N>0$, the situation is more complex as was pointed out by Hunt (1966). One has to take into account that the stabilizing Lorentz force decreases with decreasing velocity gradient in the direction of the magnetic field, i.e. with the decreasing wavenumber $k_{x}=k \cos \theta$ (see (1.9)). The $x$-independent modes with $\theta=\pi / 2$ do not generate any Lorentz forces at all. Thus, as the angle $\theta$ increases and the wave crests turn toward the flow direction, we deal with two competitive effects. The inertial energy transfer from the basic flow to the unstable wave becomes weaker, but so does the rate of the suppression by the magnetic field. For certain $N$, the second effect can have a stronger impact and a three-dimensional mode can cause the instability at smaller $R e$ than a two-dimensional one.

From the formal viewpoint, it was shown by Hunt (1966) that the information on the type of primary instability is entirely provided by the solution of (1.10) expressed in the form of the critical curve $\widehat{R e}_{c}=G(\widehat{N})$, which can be rewritten as

$$
R e_{c}=\frac{1}{\cos \theta} G(N \cos \theta) .
$$

Figure 1 shows $G(\widehat{N})$ obtained in our calculations presented in more details in the following section. According to (1.13), the stability limits to three-dimensional modes 

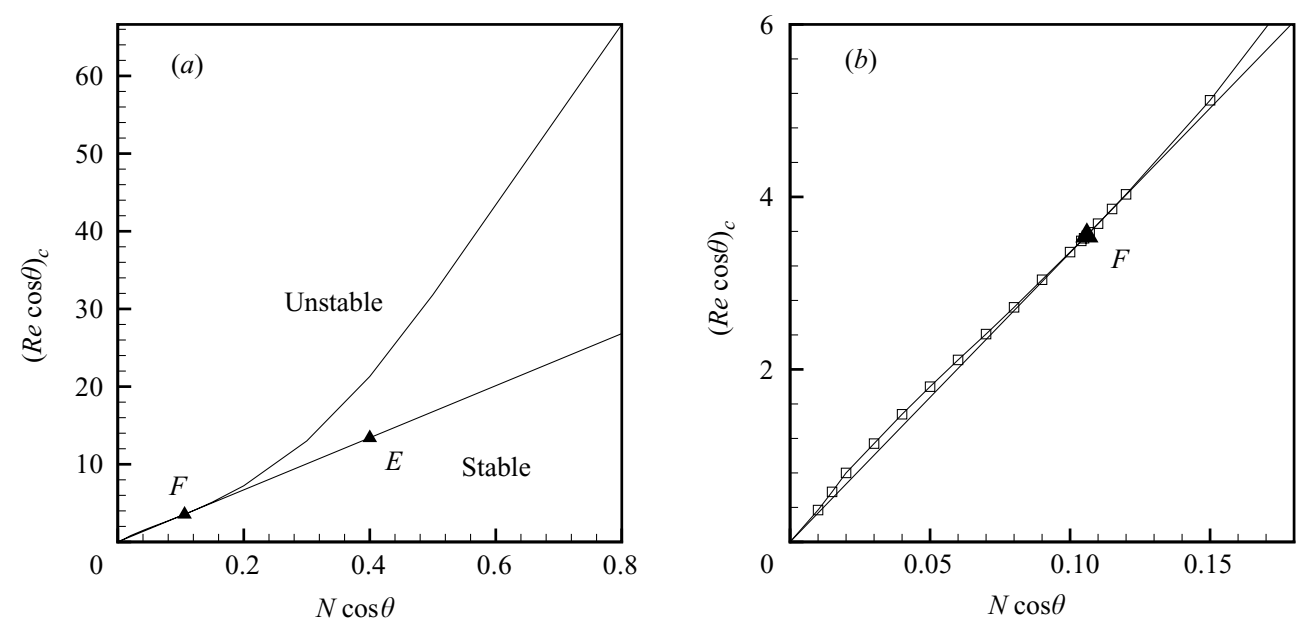

FIGURE 1. (a) Critical modified Reynolds number $\widehat{R e}$ as a function of the modified magnetic interaction parameter $\widehat{N}$. The tangent line to the curve from the origin is also shown. $(b)$ The enlarged portion of the curve at small $\widehat{N}$ and $\widehat{R e}$.

with $\theta>0$ are obtained by multiplying both abscissa and ordinate by $1 / \cos \theta$, i.e. by translating along the line drawn from the origin to the chosen point.

It can be seen that there is a special point $F$, where the tangent line drawn from the centre touches the critical curve $G(\widehat{N})$. (For better accuracy, the point $F$ was found as the minimum of the curve $\widehat{\operatorname{Re}}(\widehat{N}) / \widehat{N}$.) In our calculations, $N_{F}=0.106$ and $R e_{F}=3.55$. The abscissa $N_{F}$ corresponds to the threshold value of the magnetic interaction parameter. At $N<N_{F}$, the critical Reynolds numbers for $\theta>0$ lie above the curve. The first instability is to two-dimensional perturbations and the curve $R e=G(N)$ supplies the stability limits. At $N>N_{F}$, however, the critical points for $\theta>0$ are below the curve. The first instability is to three-dimensional disturbances. In fact, it can be shown (see figures 2 and 3 by Hunt 1966 for a simple geometrical proof) that the oblique angles of the first unstable modes are such that the stability limits lie on the tangent line $O F$ to the curve. The critical Reynolds numbers $R e_{c}$ and the oblique angles $\theta_{c}$ of the neutral modes are given by

$$
\operatorname{Re}_{c}=\operatorname{Re}_{F} \frac{N_{F}}{N}, \quad \theta_{c}=\cos ^{-1}\left(\frac{N_{F}}{N}\right) .
$$

The neutral modes themselves are defined by a single solution of the modified equation (1.10) taken at the point $\widehat{N}=N_{F}$ and $\widehat{R e}=R e_{F}$. The work of Gotoh (1971) was the first where the neutral curves of the eigenvalue problem (1.10) were actually calculated and the critical Reynolds number was determined as a function of the strength of the magnetic field and $k$. The basic velocity profile $U(z)=\tanh z$ was considered. The Hartmann number $H a=(R e N)^{1 / 2}$ representing the ratio between Lorentz and viscous forces was used as a measure of the strength of the magnetic field. It was found that the three-dimensional perturbations become more unstable than the two-dimensional ones if the field is sufficiently strong $(H a>0.52)$.

At $N_{F}=0.106$ and $R e_{F}=3.55$, we found the threshold Hartmann number to be $H a=0.61$. This is slightly different from the result of Gotoh (1971), which can be explained by the difference in the basic velocity profile. 
In this paper, we report the results of the detailed analysis of the linear instability and the resulting transition to turbulence. A free shear layer with the basic profile

$$
U(z)=\operatorname{erf}\left(\frac{\sqrt{\pi} z}{2}\right)
$$

is considered. The linear part of the analysis presented in the next section goes substantially beyond the investigation of Gotoh (1971). In addition to the type of instability (two-dimensional or three-dimensional) and the critical Reynolds numbers we investigate how the growth rate $\beta$, wavenumber $k$ and angle $\theta$ of the neutral and the fastest growing perturbations change with $N$ and $R e$. The growth and nonlinear evolution of the unstable three-dimensional disturbances and the transition to turbulence are studied in DNS calculations described in $\S 3$. Finally, $\S 4$ contains concluding remarks.

\section{Linear stability analysis}

We analyse linear stability of the basic flow (1.1) to normal modes (1.8). The standard simplifying assumption is made (see Drazin \& Reid 1981 for a review of earlier results for non-magnetic flows and Michael 1953; Drazin 1960; Hunt 1966; Abas 1969; Gotoh 1971 for the MHD case), according to which the decay of the basic flow by viscous diffusion is neglected in the linear stability analysis. One has to remember that this 'frozen basic flow' approach is strictly justified and in full agreement with the nonlinear analysis only if the typical time $\sim 1 / \beta$ of the growth of an unstable mode is much smaller than the diffusion time $\sim R e$ of the basic profile. The condition of scale separation is satisfied for the majority of the results presented below. In the cases when the two time scales are comparable (typically, at small $R e$, large $N$, and/or small $k$ ) the identified unstable modes can be viewed as related to instantaneous instability of the basic flow at a given state of its viscous decay.

The eigenvalue problem (1.10) is solved numerically using the traditional shooting approach first applied to the Orr-Sommerfeld equation by Betchov \& Szewczyk (1963). The solution is found in a layer bounded at $z=\mp L_{z}$ with $L_{z}$ chosen sufficiently large so that $U\left(\mp L_{z}\right) \approx \mp 1$ and $U^{\prime \prime}\left(\mp L_{z}\right) \approx 0$ with a high degree of accuracy and the artificial boundary conditions do not affect the solution (for the results shown, $L_{z}$ was between 15 and 25). Exponential solutions of the linear differential equation with constant coefficients obtained from (1.10) at $U\left(\mp L_{z}\right)=\mp 1$ and $U^{\prime \prime}\left(\mp L_{z}\right)=0$ are used to derive the boundary conditions for $v_{z}$. The Runge-Kutta method of the fourthorder of accuracy with automatically controlled step is employed for integration at $-L_{z} \leqslant z \leqslant L_{z}$. For better convergence of the numerical algorithm, the shooting is performed from the boundaries $z=\mp L_{z}$ to the centre $z=0$ (see Maslowe 1981). The matching conditions for $v_{z}$, its first, second and third derivatives are used to determine the eigenvalue. A similar algorithm was applied to find the eigenvalues and eigensolutions of the inviscid problem with $R e=\infty$, when only two conditions needed to be satisfied.

The shooting algorithm was designed for the search of general complex eigenvalues. However, as in the classical non-magnetic case, the eigenvalues with the largest $\beta$ were invariably found to be purely imaginary with $\omega=0$.

The results of calculations are presented in figures $2-4$. Figures $2(a)$ and $2(b)$ show the strong stabilizing effect of the magnetic field on the two-dimensional perturbations. We can see that even a moderate field suppresses the growth and reduces the range 

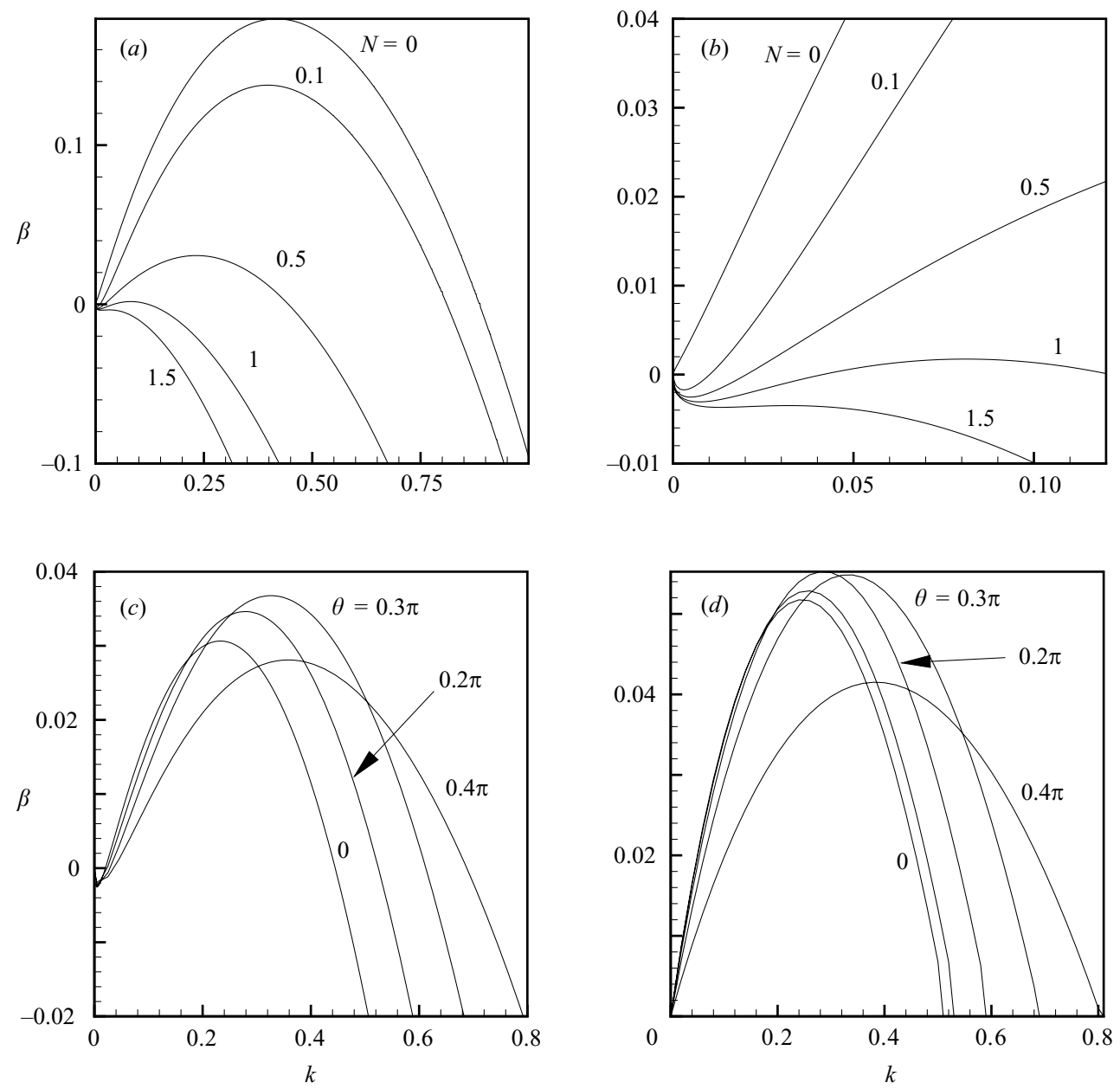

FIGURE 2. Growth rate $\beta$ vs. wavenumber $k$. (a) $R e=100, \theta=0 ;(b)$ zoom of $(a)$ to small values of $k$; (c) $R e=100, N=0.5$. (d) $R e=\infty, N=0.5$.
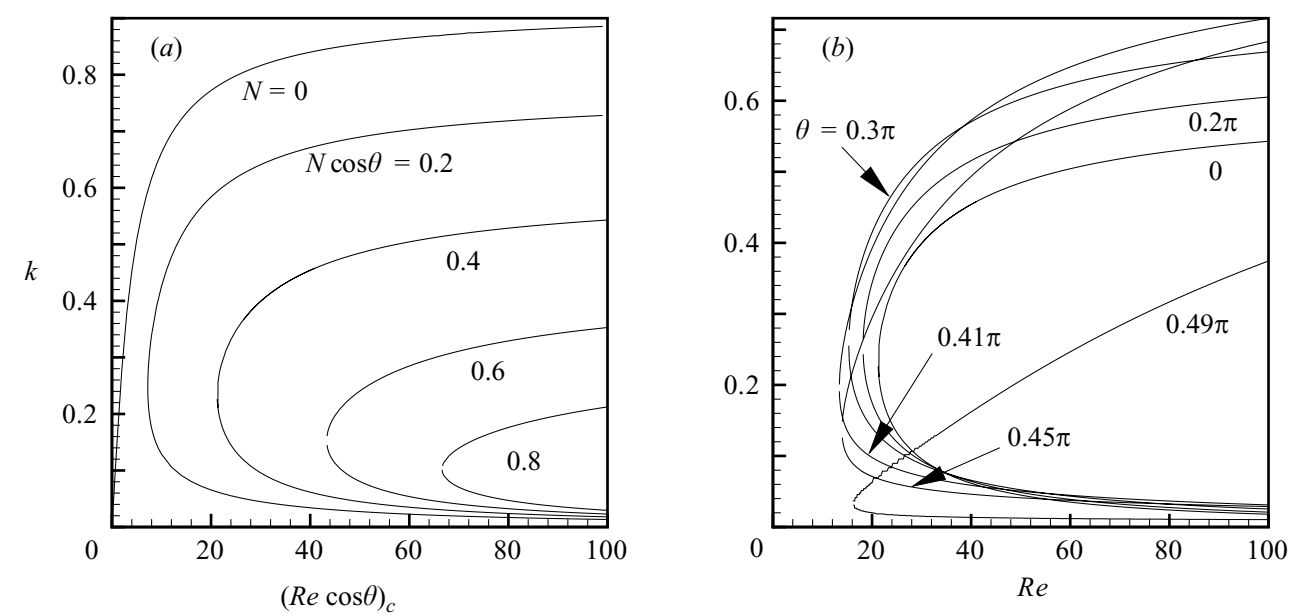

FIgURE 3. Neutral stability curves. (a) Obtained for different $\hat{N}$ by solving (1.10); (b) $N=0.4$, three-dimensional perturbation with different $\theta$. The most dangerous mode (with minimum $\left.R e_{c}\right)$ is at $\theta=0.41 \pi$. 
(a)

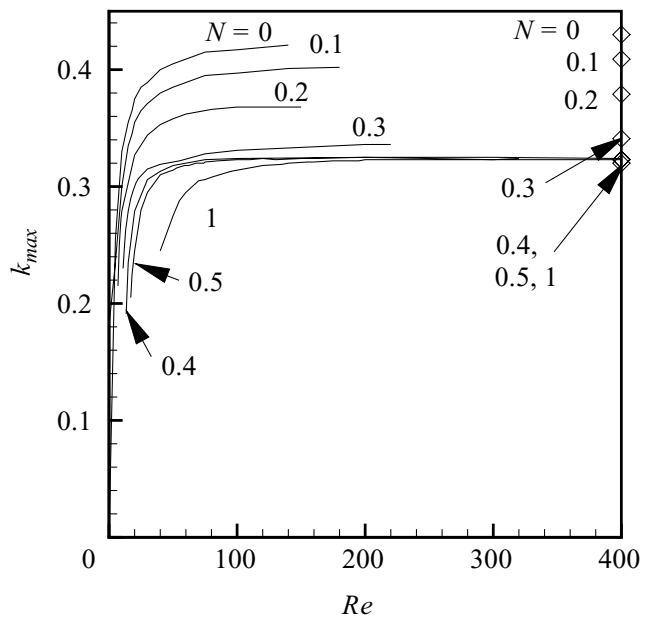

(c)

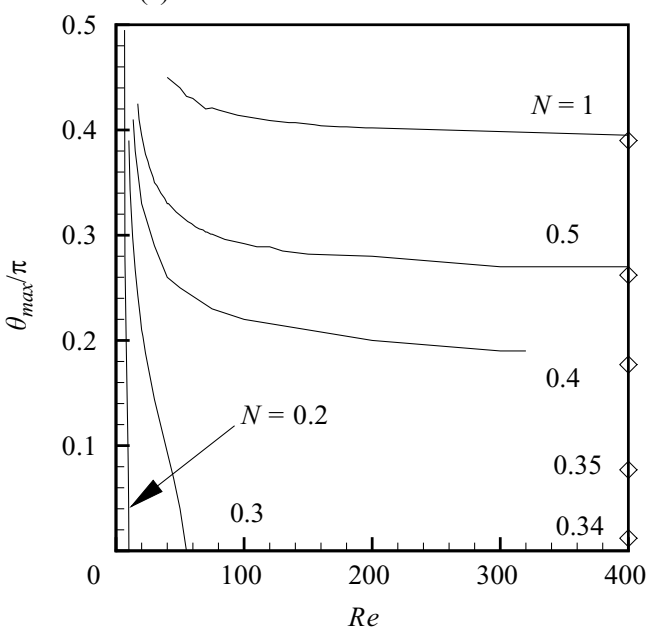

(b)

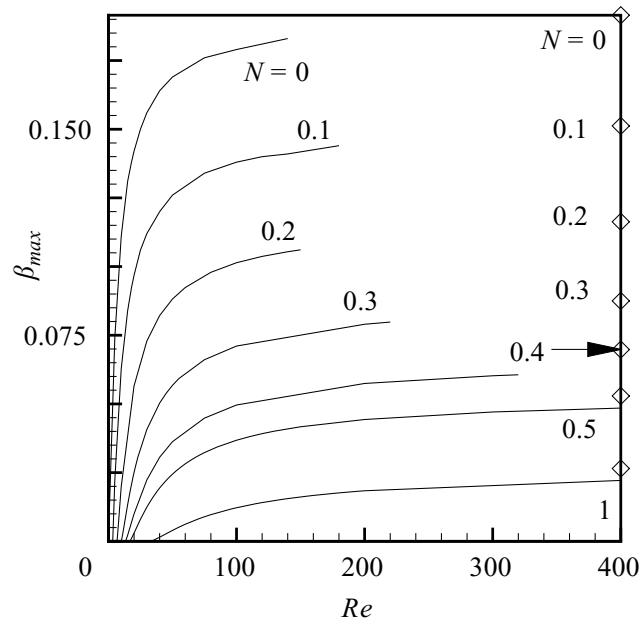

(d)

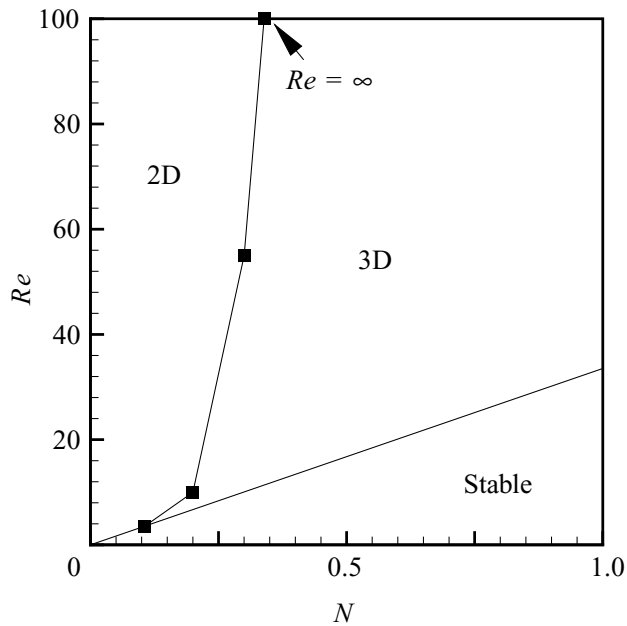

FIGURE 4. $(a-c)$ Characteristics of the fastest growing perturbations as functions of $R e$ at different $N$. The values obtained for the inviscid case $(R e=\infty)$ are marked as $\diamond$. (a) Wavenumber $k_{\max } ;(b)$ growth rate $\beta_{\max } ;(c)$ oblique angle $\theta_{\max } ;(d)$ diagram illustrating the division of the instability region into two parts with the two-dimensional (2D) or three-dimensional (3D) fastest growing disturbances.

of unstable wavenumbers. Sufficiently strong fields can completely stabilize the shear layer at finite $R e$.

It has to be stressed that the complete stabilization requires non-zero viscosity. It is shown in the inviscid two-dimensional analysis of Thess \& Zikanov (2005) that the free shear layer (1.1) cannot be completely stabilized by the magnetic field. There always exists a range of small $k$, where the flow is unstable. Such behaviour is in agreement with the intuitive picture, according to which the rate of the Joule dissipation decreases with increasing wavelength in the direction of the magnetic field, and, thus, the perturbations become less and less sensitive to the action of the magnetic field as $k \rightarrow 0$. Our results presented in figures $2(a)$ and $2(b)$ show that the picture is misleading in the case of a shear layer with finite viscosity. The magnetic 
field stabilizes the eigenmodes at both the large- $k$ and small $-k$ ends of the instability range. This was observed for two-dimensional and three-dimensional perturbations (see figure $2 b, c$ ) at all values of $R e<\infty$ and $N>0$ tested in our study. We do not have a simple physical explanation of the stabilization of long waves, but this phenomenon was detected earlier in the calculations of Gotoh (1971) and the low- $k$ analysis of Abas (1969) in the form of a second branch of the neutral curve appearing at small $k$ in the presence of a parallel magnetic field.

Typical dependence of $\beta$ on $\theta$ and $k$ for the three-dimensional disturbances is shown in figure $2(c)$. The growth rate changes slowly with the wavenumber and the oblique angle. There are substantial ranges of $k$ and $\theta$ where $\beta$ is close to $\beta_{\max }$.

Figure $2(d)$ shows the results obtained for the inviscid problem $R e=\infty$ at $N=0.5$. Curves in figures $2(c)$ and $2(d)$ are qualitatively similar, apart from the suppression of the long waves at finite Re. As discussed below, the characteristics of the unstable modes converge quickly with growing $R e$ to those of the inviscid modes.

Figure 3 presents the curves of neutral stability defined by the condition $\beta=0$. Figure 3(a) shows the critical Reynolds number $\widehat{R e}_{c}$ obtained as a function of the wavenumber $k$ through the solution of the transformed equation (1.10). As has already been illustrated in figures $2(a)$ and $2(b)$, an imposed magnetic field stabilizes the layer. A second branch of the neutral curve appearing at small $k$ is a counterpart of the second branches found by Gotoh (1971) and Abas (1969) and a manifestation of the stabilization of long waves. The critical curve $\widehat{R e}_{c}(\widehat{N})$ shown in figure 1 was obtained by minimizing the neutral curves in figure $3(a)$ over $k$.

The neutral curves corresponding to arbitrary three-dimensional perturbations can, in principal, be directly derived from figure $3(a)$. The figure, however, does not give a clear picture of the effect of the oblique angle $\theta$ on $R e_{c}$. In particular, it is difficult to say which value of $\theta$ corresponds to the most dangerous perturbation. In figure $3(b)$, we plot neutral curves for $N=0.4$ and different values of $\theta$. The critical Reynolds number $R e_{c}=13.4$ corresponds to a mode with $\theta=0.41 \pi$. The point $N=0.4, R e_{c}=13.4$ is marked as point $E$ on the straight line in figure 1(a).

Properties of the fastest growing perturbations (those with the largest growth rate $\beta_{\max }$ at given $N$ and $R e>R e_{c}$ ) are illustrated in figure 4. $\beta_{\max }$ and the corresponding wavenumber $k_{\max }$ and oblique angle $\theta_{\max }$ are shown as functions of $N$ and $R e$. The curves for $N=0$ and $N=0.1$ illustrate the behaviour typical for the case of weak magnetic field when the neutral and the fastest growing perturbations are always two-dimensional. The opposite case of strong magnetic fields when the most dangerous perturbations are invariably three-dimensional is represented by the curves for $N=0.4, N=0.5$ and $N=1$.

We found that at intermediate $N$ (curves for $N=0.2$ and $N=0.3$ in figure $4 c$ serve as examples), the fastest growing perturbations are three-dimensional only in a finite range of $\operatorname{Re}$ (at $R e<10$ if $N=0.2$ and $R e<55$ if $N=0.3$ ). At larger $R e$, the strongest growth is provided by the two-dimensional waves with $\theta=0$. We found that the boundary value of $N$ between the regions of the two-dimensional and threedimensional fastest growing perturbations is a function of Reynolds number. At the smallest $R e$ (i.e. at the stability curve), the border is at $N=N_{F}=0.106$. In the inviscid case with $R e=\infty$, the three-dimensional perturbations start to dominate when $N$ exceeds 0.339 . The situation is illustrated by the diagram in figure $4(d)$.

Figures $4(a)$ to $4(c)$ also show $k_{\max }, \beta_{\max }$ and $\theta_{\max }$ obtained for $R e=\infty$. Clear asymptotic behaviour is observed with the characteristics of the perturbations fast approaching the inviscid limits with growing $R e$. For three-dimensional perturbations 

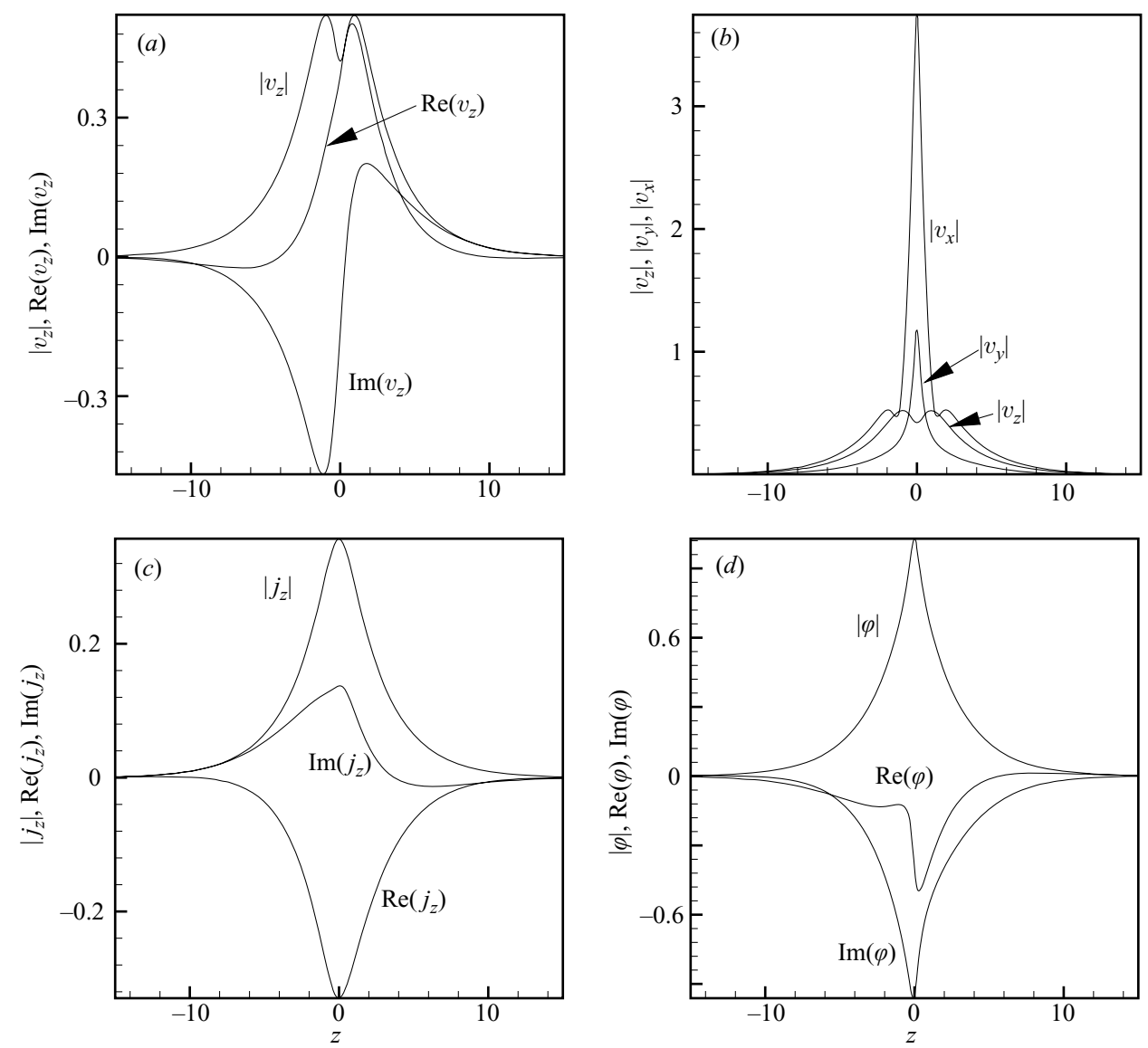

Figure 5. The eigenmode at $R e=\infty, N=0.5, k=0.323, \theta=0.262 \pi$ and $L_{z}=15$. (a) The eigenfunction $v_{z}(z)$. (b) Profiles of the absolute values of the $x$-, $y$ - and $z$-components of velocity. (c) Profile of the $z$-component of current density. (d) Profile of the electric potential $\phi$.

at moderate and large $R e$, the strength of the magnetic field affects the angle $\theta$, i.e. the orientation of the fastest growing waves, but not their wavelength. The wavenumber curves $k_{\max }(R e)$ merge at $k_{\max } \approx 0.323$ (see figure $4 a$ ).

The typical eigenfunction $v_{z}(z)$ of a three-dimensional perturbation is shown in figure $5(a)$. In the three-dimensional case, we must solve an additional boundaryvalue problem to determine the profiles of other variables and obtain the complete velocity field of the disturbance. The problem can be formulated, for example, in terms of the $z$-component of the current density $j_{z}=-\phi^{\prime}-v_{y}$ as

$$
\left(\beta+\mathrm{i} k_{x} U\right)\left(j_{z}^{\prime \prime}-k^{2} j_{z}\right)-N k_{x}^{2} j_{z}=k_{x} k_{y} U^{\prime} v_{z}+\frac{1}{R e}\left(j_{z}^{\mathrm{iv}}-2 k^{2} j_{z}^{\prime \prime}+k^{4} j_{z}\right), \quad \lim _{z \rightarrow \pm \infty} j_{z}=0 .
$$

In contrast to the Orr-Sommerfeld problem (1.9), this equation is inhomogeneous and its solution is determined by the known eigenfunction $v_{z}$. The numerical integrations of (2.1) and (1.9) were carried out simultaneously. The difficulty we met was to provide the correct boundary conditions for $j_{z}$ at $z=\overline{+} L_{z}$. Asymptotic behaviour of $j_{z}$ could not be determined from the equation itself since the inhomogeneous term of (2.1) vanishes at large $|z|$, where $U(z)=\mp 1$ and $U^{\prime}=U^{\prime \prime}=0$. Instead, we used 


\begin{tabular}{|c|c|c|c|c|c|}
\hline$\epsilon$ & $10^{-2}$ & $10^{-3}$ & $10^{-4}$ & $10^{-5}$ & $10^{-6}$ \\
\hline$\beta$ & $7.15 \times 10^{-2}$ & $5.43 \times 10^{-2}$ & $5.31 \times 10^{-2}$ & $5.29 \times 10^{-2}$ & $5.29 \times 10^{-2}$ \\
\hline
\end{tabular}
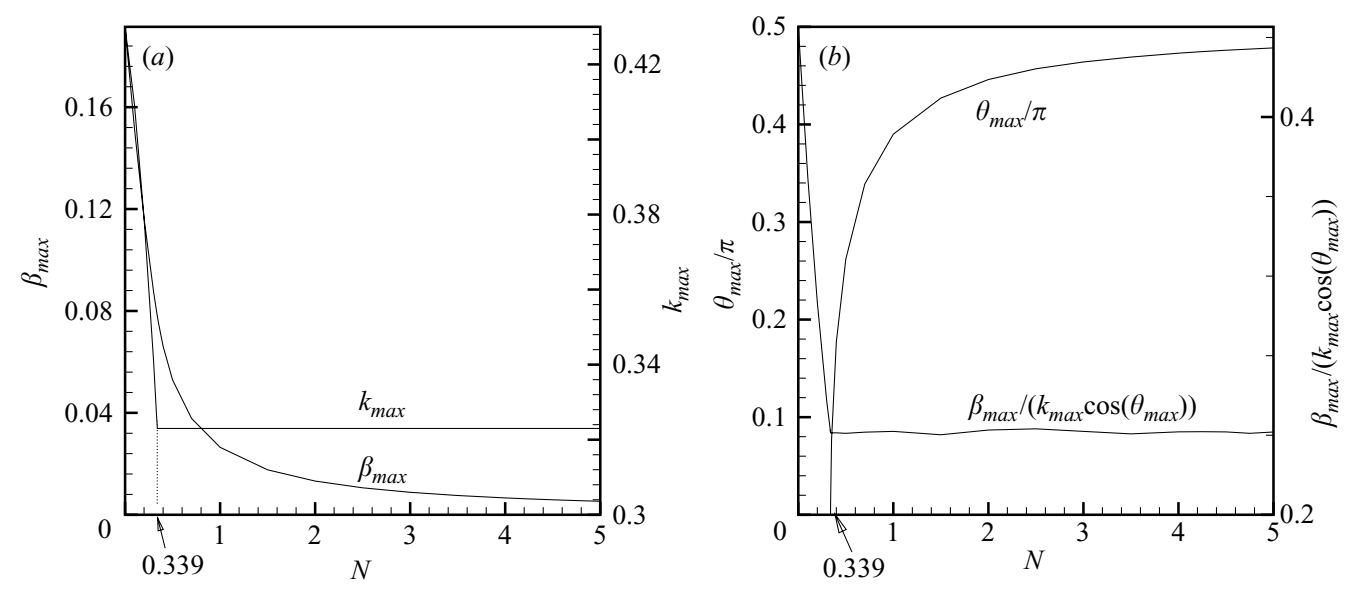

FIGURE 6. Parameters of the fastest growing perturbations at $R e=\infty$ as functions of $N$. (a) Growth rate $\beta_{\max }$ and wavenumber $k_{\max } ;(b)$ oblique angle $\theta_{\max }$ and ratio $\beta_{\max } /\left(k_{\max } \cos \theta_{\max }\right)$.

the perturbation method adding artificial perturbation terms $+\epsilon j_{z}$ and $+\epsilon v_{z}$ to the right-hand sides of (1.9) and (2.1), respectively. Here, $\epsilon$ is the formal parameter, and the correct solution is obtained in the limit of $\epsilon=0$. The calculations showed the efficiency and accuracy of the method. We found fast convergence at $\epsilon \rightarrow 0$, both for eigenvalues and eigenfunctions (see table 1 for an illustration). The $x$ - and $y$ components of the velocity are shown in figure $5(b)$, while figures $5(c)$ and $5(d)$ show the $z$-component of the current density and the electrical potential.

Visual inspection of the velocity fields of three-dimensional eigenmodes (see figure $7 a$ and accompanying discussion in the next section) reveals a structure reminiscent of the structure of a usual horizontal Kelvin-Helmholtz roll with the only distinction being that its axis is not perpendicular to the direction of the flow, but is inclined to it at an angle $(\pi / 2-\theta)$.

It was shown in figure 4 that the characteristics of the fastest growing modes quickly converge to the characteristics of the inviscid modes as $R e$ grows. In order to investigate the effect of the magnetic field in the asymptotic limit of low viscosity, we solved the eigenvalue problem at $0 \leqslant N \leqslant 5$ and $R e=\infty$. The results presented in figure 6 demonstrate remarkable behaviour. In order to understand it better, we have to return to the modified Orr-Sommerfeld equation (1.10) and write its solution, the eigenvalue $\widehat{\beta}$ (we assume that the oscillation frequency $\omega$ is zero) as

$$
\widehat{\beta}=H(k, \widehat{N})),
$$

where $H$ is some function. The relation can be rewritten as

$$
\beta=k \cos \theta H(k, N \cos \theta) .
$$


The maximum growth rate $\beta_{\max }$ is the maximum of the right-hand side of (2.3) taken over all possible values of $k$ and $\theta$

$$
\beta_{\max }=\max _{k, \theta}[k \cos \theta H(k, N \cos \theta)]=k_{\max } \cos \theta_{\max } H\left(k_{\max }, N \cos \theta_{\max }\right) .
$$

Growth of $N$ changes one of the arguments of the function $H$, but also affects $k_{\max }$ and $\theta_{\max }$. In the case of two-dimensional perturbations at $N<0.339$ the situation is simple. Increasing $N$ leads to a decrease of both $\beta_{\max }$ and $k_{\max }$ (stronger magnetic fields result in slower growth and longer waves). It was shown elsewhere (Thess \& Zikanov 2005) that this behaviour extends continuously to arbitrary large $N$.

In the case of the three-dimensional fastest growing perturbations, the effect of the magnetic field is completely different. We can see in figure 6 that $k_{\max }$ is constant (equal to 0.323 ) at all $N>0.339$. The growth rate $\beta_{\max }$ and the oblique angle $\theta_{\max }$ change with $N$ in such a way that the combination $\beta_{\max } / k_{\max } \cos \theta_{\max }$ is constant and approximately equal to 0.241 . This means that $H\left(k_{\max }, N \cos \theta_{\max }\right)=\mathrm{const}=0.241$ and, since $k_{\max }$ does not change, $\widehat{N}=N \cos \theta_{\max }$ has a constant value equal to the critical value at which $\theta_{\max }$ departs from zero, i.e. 0.339 .

We see that the behaviour of the fastest growing three-dimensional modes is similar to the behaviour found for the neutral modes by Hunt (1966). All these modes at different $N$ are, in fact, given by a single solution of the modified Orr-Sommerfeld equation obtained, this time, at $\widehat{N}=0.339$. This was confirmed by the analysis of the eigenfunction $v_{z}(z)$, which showed the profile independent of $N$. The conclusion can be made that the increasing strength of the magnetic field does not affect the dimensions or spatial shape of the fastest growing Kelvin-Helmholtz waves. Its only effect is the increase of the oblique angle according to

$$
\theta_{\max }=\cos ^{-1}\left(\frac{0.339}{N}\right) \text {. }
$$

Other features of the asymptotic behaviour of the three-dimensional perturbations are easy to see. The growth rate is

$$
\beta_{\text {max }}=0.241 k_{\max } \cos \theta_{\max }=\frac{0.026}{N},
$$

and the parallel and perpendicular wavelengths are

$$
\ell_{x}=\frac{2 \pi}{k_{\max } \cos \theta_{\max }}=57.4 N, \quad \ell_{y}=\frac{2 \pi}{k_{\max } \sin \theta_{\max }}=\frac{\ell_{x}}{\tan \theta_{\max }}=\frac{19.5 N}{\left(N^{2}-0.339^{2}\right)^{1 / 2}} .
$$

At finite $R e$, the behaviour does not exactly follow the asymptotic relations (2.5)(2.7), but, as illustrated in figure 4 , is fairly close to them even at moderate values of Re.

\section{Nonlinear evolution and transition to turbulence}

In this section, we report the results of numerical experiments conducted to investigate the transition to turbulence triggered by the growing oblique waves. A temporally evolving mixing layer affected by an imposed parallel magnetic field is considered. We apply the method of direct numerical simulations and try to reproduce the entire transition. Another promising approach, namely the nonlinear stability analysis aiming to predict the secondary pattern formation is not pursued in our paper. 
The numerical method is based on the fully de-aliased Fourier pseudo-spectral approach in the horizontal directions and the second-order finite-difference scheme in the normal direction. A more detailed description of the numerical algorithm is available in Zikanov, Slinn \& Dhanak (2004). The finite-difference grid is non-uniform with clustering in the area of strong basic shear. The clustering is achieved by applying the mapping

$$
z=L_{z} \frac{\sinh (A(\zeta-0.5))}{\sinh (0.5 A)},
$$

where $0 \leqslant \zeta \leqslant 1$, the computational domain is $-L_{z} \leqslant z \leqslant L_{z}$, and the stretching coefficient is $A=4$. The boundary conditions at $z= \pm L_{z}$ are those of a stress-free impermeable wall for the velocity and zero normal derivative for the electric potential. The periodic boundary conditions are assumed in the streamwise $(x)$ and spanwise (y) directions. For the results shown below, the vertical size of the domain is $2 L_{z}=40$. Test runs with $2 L_{z}=50$ showed that such values of $L_{z}$ secure negligible influence of the artificial boundary conditions on the solution. In the $x$ - and $y$-directions, the computational domain is rectangular with the dimensions $L_{x}=2 \pi / k_{x, \text { max }}$ and $L_{y}=2 \pi / k_{y, \max }$, where $k_{x, \max }$ and $k_{y, \max }$ are the components of the wavenumber vector corresponding to the most unstable perturbation for given parameters.

The time integration is performed using the time-splitting projection algorithm. Non-linear, viscous and electromagnetic terms of the momentum equation are integrated explicitly using the Adams-Bashforth scheme of the third-order of accuracy with adjustable time step (Zikanov et al. 2004). The Poisson equations for pressure $p$ and electric potential $\phi$ are solved using the Fourier transform in the $x$ - and $y$-directions and the double-sweep algorithm in the $z$-direction.

The results presented below were calculated with the numerical resolution based on $256 \times 256$ Fourier modes and 300 vertical grid points. Test runs conducted with the resolution $128 \times 128 \times 390$ showed no significant differences. The ' $3 / 2$ rule' was used to remove aliasing errors, which reduced the true resolution in the $x$ and $y$ directions by one-third. The algorithm was parallelized and typically executed on 8 to 20 CPUs. As a test of the numerical model, the results of Metcalfe et al. (1987) for the transition in a free shear layer with $R e=400$ and $N=0$ were reproduced with good agreement.

Transition to turbulence in a free shear layer is a complex process, which can proceed along many different paths and is affected by many different mechanisms. Extensive earlier investigations of the non-magnetic case (see e.g. Metcalfe et al. 1987; Rogers \& Moser 1992, 1993) identified the mechanisms and showed that their relative importance was largely determined by the composition of the initial perturbations added to the basic flow and the features of the model such as the horizontal size of the computational domain (one or several wavelengths), choice between temporal or spatial evolution of the layer, etc. In real flows with the initial perturbations being some form of noise, the transition is likely to be a result of superposition of several mechanisms.

In the flow with magnetic field, the scenario of transition is likely to be equally complex and dependent on multiple factors. Our intention in this study is not to reveal and document the process in all its complexity. The goal is rather to conduct the first analysis of a novel transition scenario based on the growth of the unstable oblique waves.

In the simulations, we use $R e=500$ in all runs, $N=0.5, N=1.0$, and, for comparison, $N=0$. The initial conditions are superpositions of the basic flow (1.15), the eigensolution $\boldsymbol{v}_{1}$ for the fastest growing modes, and the random velocity field $\boldsymbol{v}_{2}$,

$$
\boldsymbol{v}(x, y, z, 0)=\boldsymbol{U}(z)+A_{1} \boldsymbol{v}_{1}+A_{2} \boldsymbol{v}_{2} .
$$


(a)

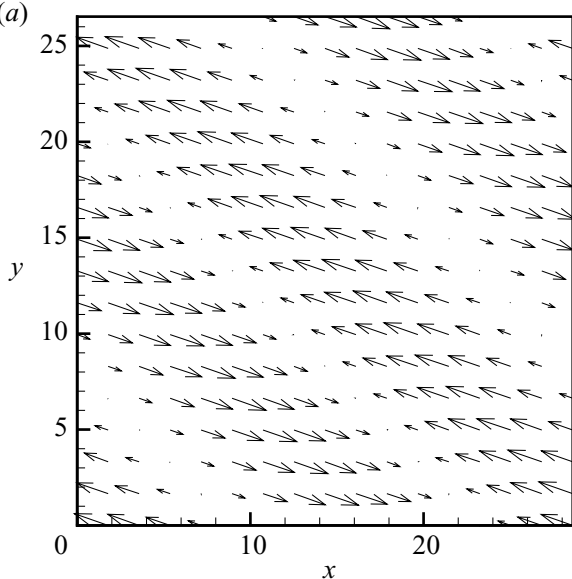

(b)

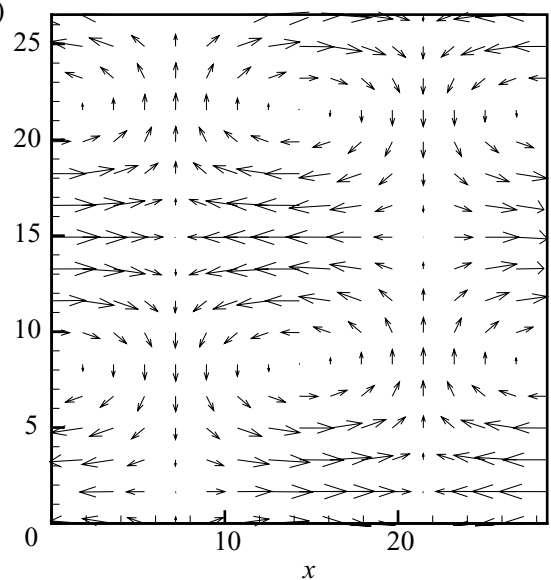

FIGURE 7. Horizontal projections of the initial velocity field in the cross-section at $z=0$. $N=0.5, R e=500, k_{\max }=0.323$, and $\theta_{\max }=0.262 \pi$. (a) one-wave and $(b)$ two-wave disturbance.

The amplitudes $A_{1}$ and $A_{2}$ are chosen so that the initial energies of the eigensolution and random noise are, respectively, $10^{-4}$ and $10^{-6}$ in non-dimensional units. The parameters of the eigensolutions are $\theta_{\max }=0$ and $k_{\max }=0.43$ for $N=0, \theta_{\max }=0.262 \pi$ and $k_{\max }=0.323$ for $N=0.5$, and $\theta_{\max }=0.39 \pi$ and $k_{\max }=0.323$ for $N=1$.

In the case of oblique waves, the symmetry of the problem results in equal linear growth rates of the two symmetric waves with positive and negative oblique angles, i.e. with $\boldsymbol{k}=\left(k_{x}, k_{y}\right)$ and $\boldsymbol{k}=\left(k_{x},-k_{y}\right)$, and of any their linear combination. We performed simulations for two such combinations in the initial conditions (3.2), a single wave with the oblique angle $\theta_{\max }>0$ and a superposition of two waves with equal amplitudes and angles $\pm \theta_{\max }$ (figure 7 ). The initial energies of both types of disturbance are equal.

We start with the simulations performed at $N=0.5$ and the initial conditions (3.2) consisting of one or two oblique waves. Figure 8 shows the evolution of the total energy of the flow $E$, the energy of the three-dimensional perturbations of the mean flow $E^{\prime}$, and the rates of viscous and magnetic dissipation $\epsilon$ and $\mu$ calculated as

$$
\left.\begin{array}{r}
E=\frac{1}{V} \int_{V}\left(v_{x}^{2}+v_{y}^{2}+v_{z}^{2}\right) \mathrm{d} V, \quad E^{\prime}=\frac{1}{V} \int_{V}\left(\left(v_{x}-\left\langle v_{x}\right\rangle\right)^{2}+v_{y}^{2}+v_{z}^{2}\right) \mathrm{d} V, \\
\epsilon=\frac{1}{V \operatorname{Re}} \int_{V}\left(\boldsymbol{v} \cdot \nabla^{2} \boldsymbol{v}\right) \mathrm{d} V, \quad \mu=\frac{N}{V} \int_{V}((\boldsymbol{j} \times \boldsymbol{B}) \cdot \boldsymbol{v}) \mathrm{d} V .
\end{array}\right\}
$$

Here $\langle\ldots\rangle$ stands for horizontal averaging, $\langle f\rangle=\left(1 /\left(L_{x} L_{y}\right)\right) \int_{0}^{L_{y}} \int_{0}^{L_{x}} f \mathrm{~d} x \mathrm{~d} y$, and $V$ is the volume of the computational domain. Curves of $E$ and $\epsilon$ corresponding to the purely diffusive decay of the unperturbed basic flow are shown for comparison.

The initial development of both types of perturbation is dominated by nearly linear growth of the eigenmodes as illustrated by the closeness of the perturbation energy curves in figure $8(b)$ to the exponential curve $\sim \exp (2 \times 0.0529 t)$, where $\beta=0.0529$ is the growth rate predicted by the linear analysis. There is a small discrepancy between the linear and nonlinear growth rates, even at the earliest stages of the evolution. This can be explained by the viscous diffusion of the mean flow and by the effect of random noise. 

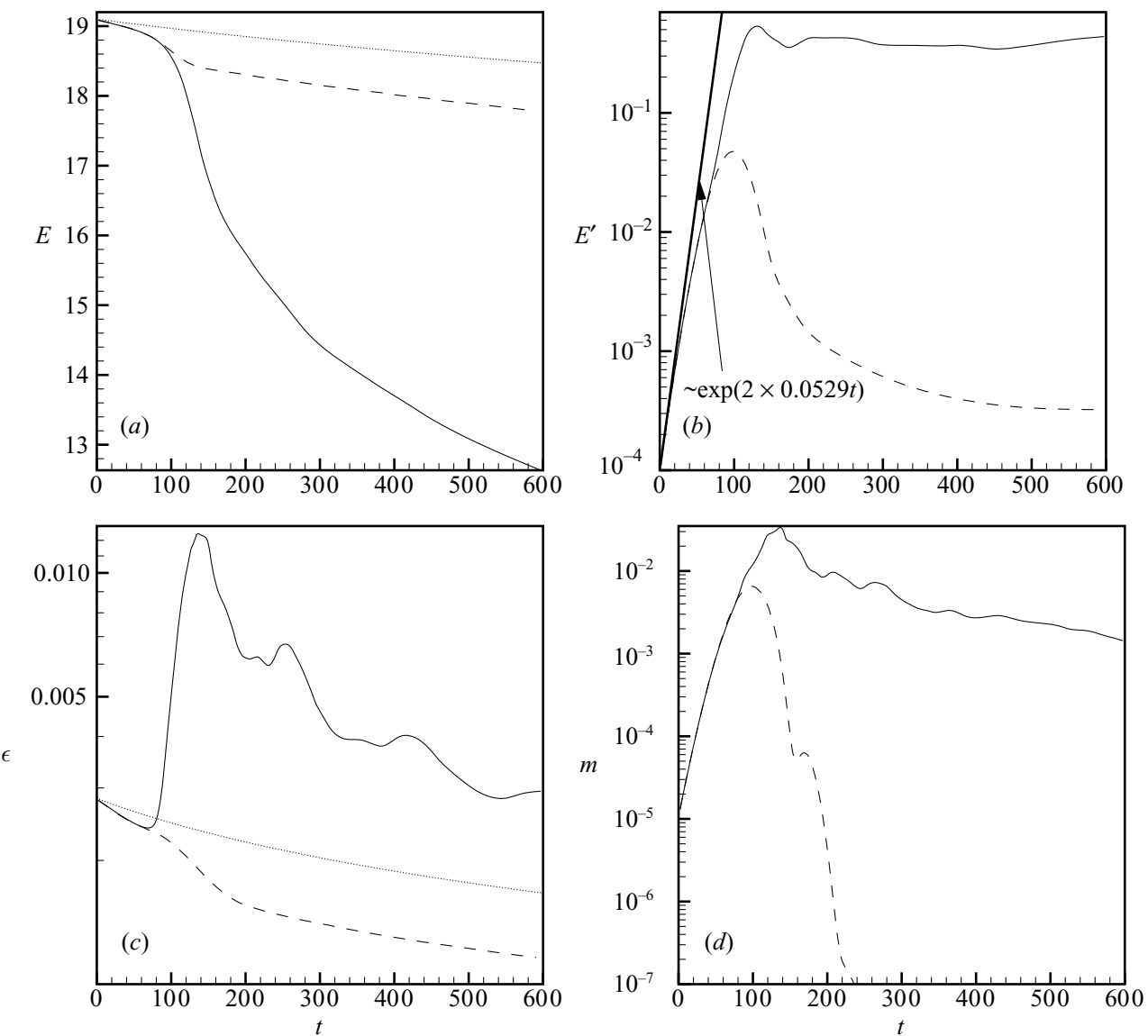

Figure 8. Evolution of total energy $(a)$, energy of three-dimensional perturbations of the mean flow $(b)$, viscous $(c)$ and magnetic $(d)$ dissipation rates. The transition at $N=0.5$, $R e=500, k_{\max }=0.323$, and $\theta_{\max }=0.262 \pi$ is triggered by a single oblique wave (-- $)$ or by a superposition of two symmetric waves (-). Dotted lines in $(a)$ and $(c)$ correspond to the diffusion of the unperturbed basic velocity profile.

It can be seen in figure 8 that there are strong differences in the behaviour of the one-wave and two-wave solutions at the nonlinear and turbulent stages. The flow initiated by two waves is characterized by much larger (about an order of magnitude) peak energy of the perturbations (see figure $8 b$ ) and stronger viscous and magnetic dissipations (see figure $8 c, d$ ). Its development leads to much stronger mixing of the mean flow as illustrated by the drop in the total energy in figure $8(a)$. The three-dimensional perturbations evolving from the one-wave initial conditions are much weaker, although undoubtedly present, as can be seen by the growth of $E^{\prime}$ in figure $8(b)$, and faster than the diffusive decrease of $E$ in figure $8(a)$. A feature of this flow (figure $8 c, d$ ) is that the suppression of the perturbations occurs in a relatively short time period characterized by a sharp peak in the magnetic dissipation curve. The viscous dissipation rate is much weaker. Furthermore, it can be noted that the main contribution to $\epsilon$ shown in figure $8(c)$ is provided by the dissipation of the mean flow.

The difference between the one-wave and two-wave solutions is due to different types of secondary instability and different ways, in which turbulence appears in the flows. This becomes evident after we consider the evolution of the spatial structure of 
(a)

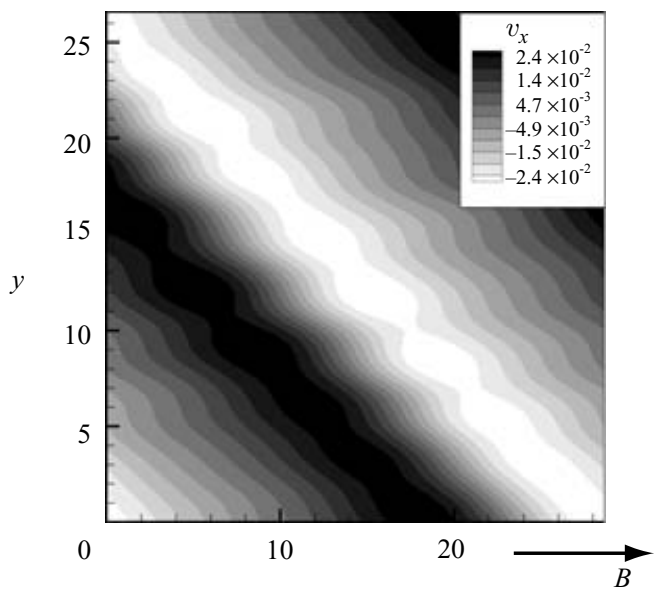

(c)

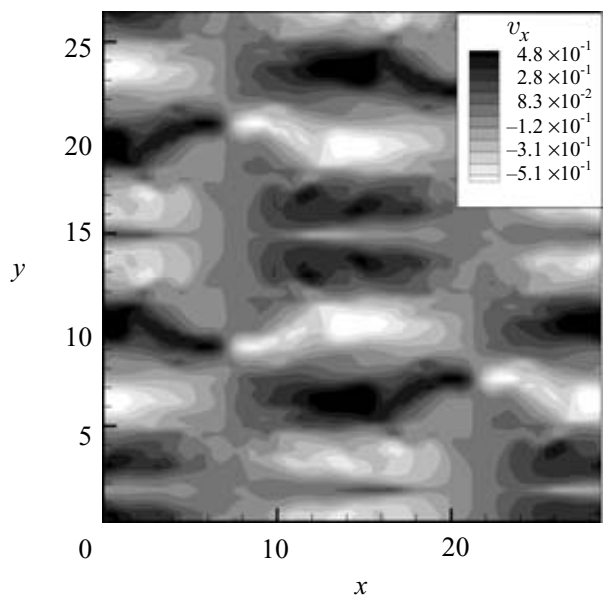

(b)

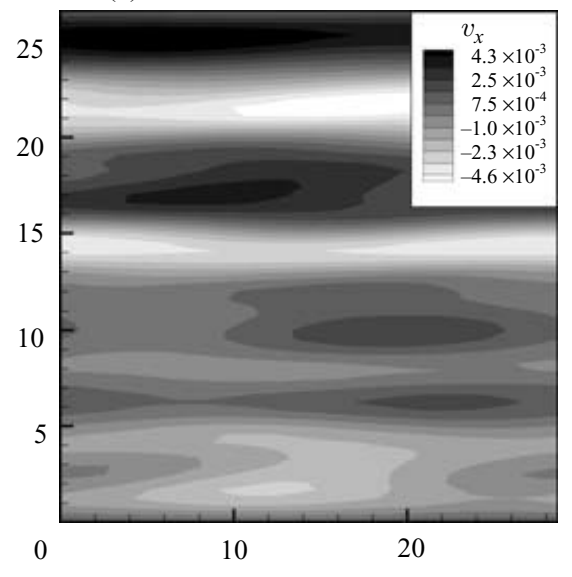

$(d)$

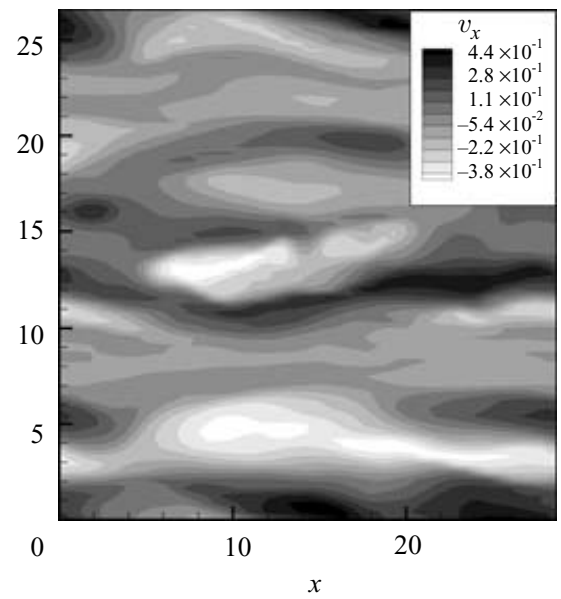

FIGURE 9. Contours of streamwise velocity (in the $z=0$ plane) are shown for solutions at $R e=500, N=0.5, k_{\max }=0.323$, and $\theta_{\max }=0.262 \pi$ obtained with one wave $(a, b)$ and two waves $(c, d)$ in the initial conditions. The snapshots are taken at the stages of the breakup of the growing waves $(a, c)$ and decay of turbulence $(b, d) .(a) t=162,(b) 251,(c) 153,(d) 327$.

the flow illustrated by the contours of streamwise velocity (figure 9), two-dimensional energy power spectra (figure 10), and the isosurfaces of the amplitude of perturbation vorticity $\omega=|\nabla \times(\boldsymbol{v}-\langle\boldsymbol{v}\rangle)|$ (figure 11). The spectra are calculated using the Fourier transform of the velocity field in the plane $z=0$. The plots show the energy $E\left(q_{x}, q_{y}\right)$ of the Fourier modes with the wavenumber vectors $\boldsymbol{k}=\left(q_{x} k_{x}, q_{y} k_{y}\right)$ as a function of integers $q_{x}$ and $q_{y}$. Only half of the wavenumber plane at $q_{x} \geqslant 0$ is depicted. The left-hand half-plane, which is not shown, corresponds to the complex-conjugate Fourier modes. Considering the spectra, we have to take into account that only the modes with even $q_{x}+q_{y}$ can appear in the result of the interaction between the basic flow, linear unstable modes, and their subharmonics. It is only because of the smallamplitude noise in the initial conditions that the spectra are filled continuously at the late turbulent stages of the flow evolution.

In the case of the one-wave solution, the early stages of nonlinear development are characterized by the growth and deformation of parallel oblique rolls. This can be 
(a)

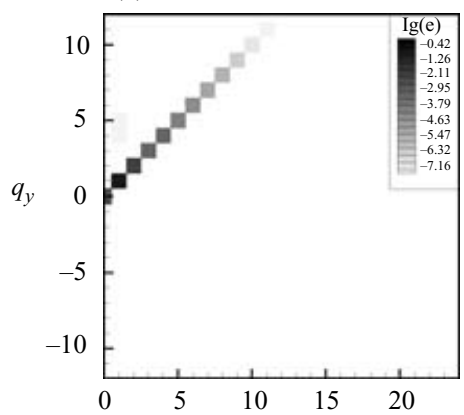

(d)

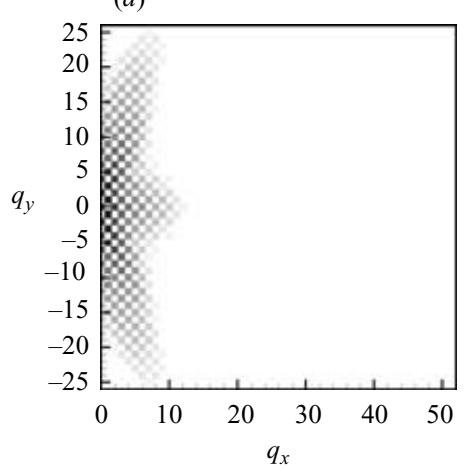

(b)

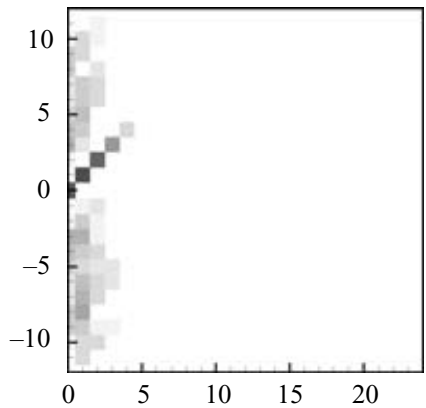

(e)

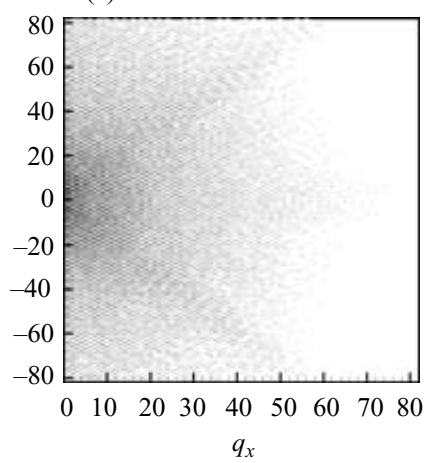

(c)
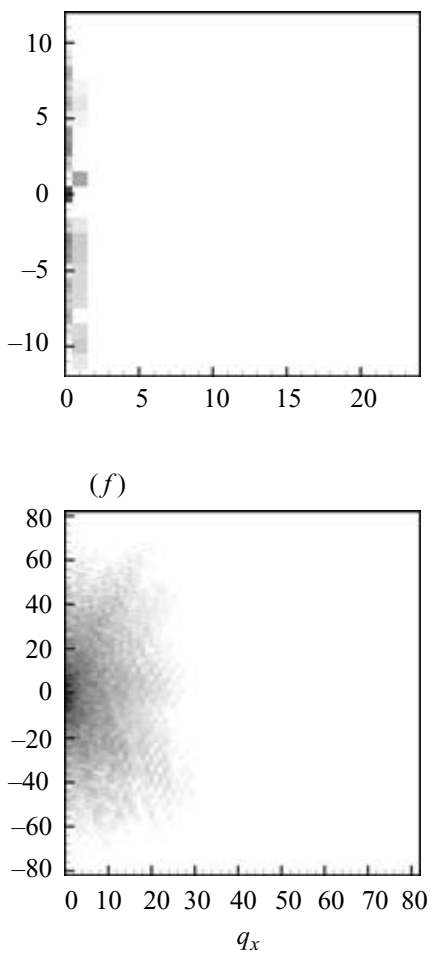

FIGURE 10. Power energy spectra of velocity fields at $z=0$ for different moments of time for solutions at $R e=500, N=0.5, k_{\max }=0.323$ and $\theta_{\max }=0.262 \pi$ obtained with one wave $(a-c)$ and two waves $(d-f)$ in the initial conditions. The energy levels are the same in all pictures. The modes with energy less than $10^{-8}$ are blanked. (a) $t=79.0,(b)$ 162, (c) 251, (d) 78.7, (e) $153,(f) 327$.

seen in the vorticity field in figure $11(a)$. We can also see that the dominant modes of the energy spectrum in figure $10(a)$ are the principal unstable mode $(1,1)$ and its subharmonics. The main element of the further development of the flow is the secondary instability of the oblique rolls. It starts in the form of spanwise waves (see figures $9 a$ and $11 b$ ) and quickly leads to breakup of the rolls and appearance of weak turbulence around the midplane of the layer (see figures $9 b$ and $11 c$ ). The magnetic field plays a decisive role in the evolution by suppressing the turbulence and transforming it into anisotropic form. It can be seen that the turbulent eddies are elongated along the magnetic field lines. The decaying turbulent flow shown in figures $9(b)$ and $11(c)$ is only slightly non-uniform in the $x$-direction. The effect can also be seen in the power spectra in figure $10(c)$. High- $q_{x}$ modes are suppressed by the magnetic field and the spectrum takes the anisotropic form typical for MHD turbulence at moderate to high $N$ (Zikanov \& Thess 1998; Vorobev et al. 2005).

The flow evolution in the case of the one-wave initial conditions has some features reminiscent of the non-magnetic scenario (see, for example, Metcalfe et al. 1987; Rogers \& Moser 1992, 1993). The wavelength of the secondary waves in figure 9(a) is much smaller than the wavelength of the unstable modes of the linear stability problem for the basic flow. We can assume that the mechanism leading to these waves is the elliptic instability of strained vortices (Kerswell 2002). Vortical structures 

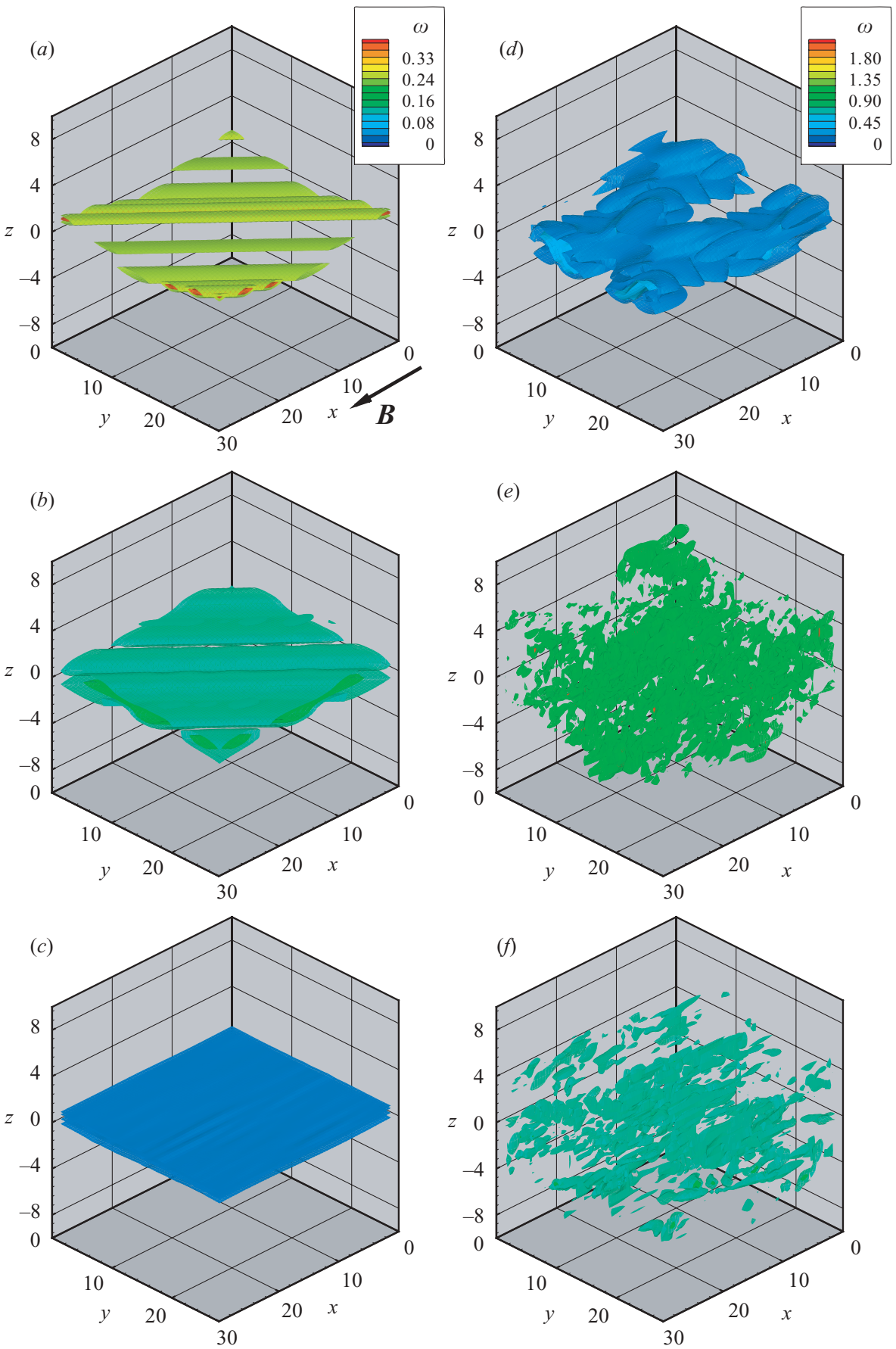

FIGURE 11. Isosurfaces of the amplitude of perturbation vorticity $\omega=|\nabla \times(\boldsymbol{v}-\langle\boldsymbol{v}\rangle)|$ are shown for different moments of time for solutions at $R e=500, N=0.5, k_{\max }=0.323$ and $\theta_{\max }=0.262 \pi$ obtained with one wave $(a-c)$ and two waves $(d-f)$ in the initial conditions. The isosurfaces are drawn at $\omega=0.5 \omega_{\max }$ and $\omega=0.75 \omega_{\max }$ in the case of the one-wave solution and at $\omega=0.25 \omega_{\max }$ and $\omega=0.5 \omega_{\max }$ in the case of the two-wave solution. Note the different level scales used in $(a-c)$ and $(d-f)$. (a) $t=79.0, \omega_{\max }=0.504 ;(b)$ 162, 0.222; (c) 251, 0.059; (d) 78.7, 0.792; (e) 153, 4.13; (f) 327, 2.49. 
(a)

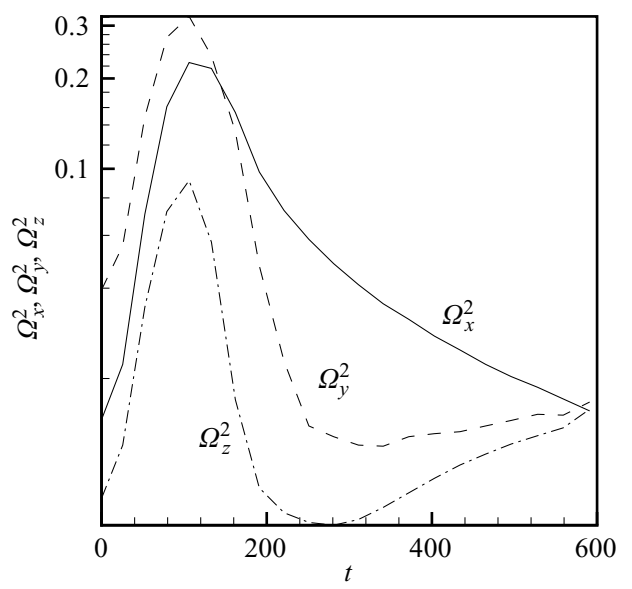

(b)

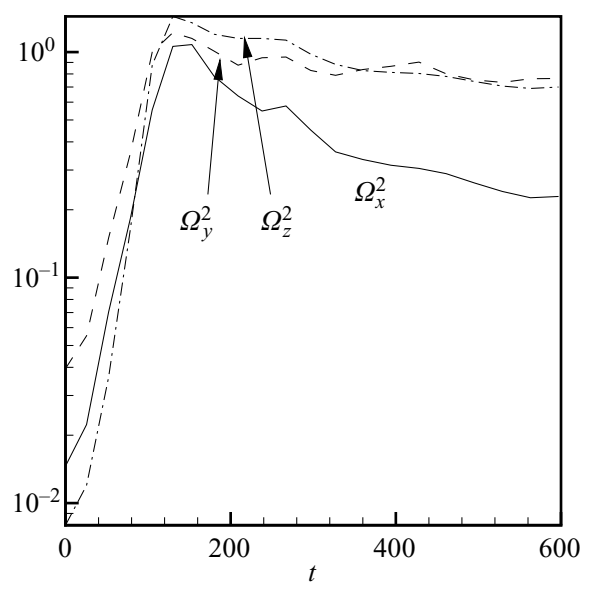

Figure 12. Volume averages of the squares of the $x(-), y(---)$ and $z(-\cdot-)$ components of the perturbation vorticity at $R e=500, N=0.5, k_{\max }=0.323$ and $\theta_{\max }=0.262 \pi$ obtained with $(a)$ one wave and $(b)$ two waves in the initial conditions.

similar to the 'ribs' in the 'braid' regions (Metcalfe et al. 1987) were observed at some stages of the flow evolution. There are also substantial differences between the magnetic and non-magnetic cases because of the suppression and re-orientation of the secondary structures by the magnetic field and the fact that in the magnetic case the rolls are not perpendicular to the mean flow. A detailed study of the transition that would provide a better understanding of these similarities and differences would require simulations with the three-dimensional perturbations of special form added to the growing eigensolution. We do not pursue this path and limit our study to a noise-induced secondary instability because, as discussed below, there is a more powerful and more interesting transition scenario unique to the MHD case.

The evolution of the flow starting with the two-wave initial conditions is completely different from the evolution described above. The principal reason is that the twowave flow is essentially three-dimensional from the very beginning. It can be seen in figure $7(b)$ that superposition of two symmetric eigenmodes results in a cellular pattern of perturbation velocity. Our simulations show that this pattern is much less capable of long-term existence as a coherent structure than the one-wave oblique rolls. The process of its disintegration starts soon after the growing perturbations enter the nonlinear phase. Even at the earlier stage of the flow development (figure 10d), non-negligible energy is present in the Fourier modes outside the main subharmonic branches $\gamma(1,1), \gamma(1,-1), \gamma(1,0)$, where $\gamma$ is an integer number. At a later time, the excitation grows in amplitude and spreads over a wide range of the wavenumbers (see figure 10e). (The two-wave simulation described here was as demanding with regard to the required horizontal numerical resolution as the simulation of the nonmagnetic transition at $N=0$. We found that $256 \times 256$ Fourier modes were required to represent accurately the perturbations at the peak of their energy.)

It can be seen in figures $9(c), 10(e)$, and 11(e) that the flow is strongly turbulent at $t>100$. Although remnants of the cellular pattern are still present, substantial portion of the energy of perturbations is in the small-scale chaotic fluctuations. The perturbation vorticity grows as illustrated in figure 12 by the volume averages of the squares of its components $\Omega_{i}^{2}=V^{-1} \int_{V}(\nabla \times v-\langle\nabla \times v))_{i}^{2} \mathrm{~d} V$. 
The subsequent development of the layer is characterized by the decay of turbulence under the combined action of the viscous and magnetic dissipations and by the vertical diffusion of turbulence. It can be seen in figure $11(f)$ that the region of noticeable turbulence levels spreads vertically. Substantial vorticity appears at $|z|$ up to 10 , where the basic flow has virtually zero shear. The traces of the cellular pattern disappear completely (see figures $9 d$ and $11 f$ ).

As expected, in addition to the anisotropy imposed by the mean shear, the magnetic field generates the typical anisotropy of gradients (elongation of the flow structures in the direction of $\boldsymbol{B}$ ) in the decaying turbulent flow. The degree of anisotropy of the two-wave flow is lower than in the case of the one-wave solution. This is confirmed by visual inspection of velocity and vorticity fields in figures $9(b, d)$ and $11(c, f)$ and spectra in figure $10(c, f)$. A simple explanation is that the effective parameter $N$ for the interaction between the magnetic field and the turbulent fluctuations should be based on the characteristics of the perturbations instead of the characteristics of the basic flow. For example, we can substitute the r.m.s. fluctuation velocity and the integral length scale in place of $U_{0}$ and $L$ in (1.6). Alternatively, we can use the vorticity-based definition

$$
N_{\text {vort }}=\frac{\sigma B^{2}}{\rho \omega_{0}},
$$

where $\omega_{0}$ is a typical value of vorticity. With both definitions, the effective interaction parameter is smaller in the two-wave flow, which agrees with the lower degree of anisotropy.

The instability and turbulence dramatically increase the degree of mixing in a free shear layer. In order to quantify this effect, we calculate the mean velocity profile $\left\langle v_{x}\right\rangle(z, t)$ and the $95 \%$ thickness of the mixing layer defined as the minimum distance from the midplane beyond which the deviation of the velocity magnitude from its far-field value is less than $5 \%$

$$
\delta_{95}(t)=\min \left\{z^{*} ;\left|v\left(x, y,|z|>z^{*}, t\right)\right|>0.95\right\} .
$$

The results obtained in the one-wave and two-wave solutions are presented in figure 13. For comparison, we also show the mean velocity profile of a purely diffusive flow (the dashed lines) and the results of the non-magnetic simulation. The evolution of the non-magnetic flow follows the classical path of growth and saturation of two-dimensional billows and their subsequent three-dimensional breakdown around $t=100$.

The suppression of turbulence by the magnetic field should, in general, lead to a decreased thickness of the mixing layer. It can be seen in figure $13(b)$ that this is true for the one-wave MHD flow. $\delta_{95}$ grows to only about 2.5 and settles at almost diffusive growth after $t \approx 100$. Remarkably, this moment corresponds to an early stage of the evolution of the flow structure illustrated in figures 9-11, approximately to the phase of the spanwise instability of the oblique rolls. We see that the convective mixing in the one-wave solution is primarily due to the growth of these rolls. Their subsequent breakdown occurs against a background of constant decline of the perturbation energy (see figure 8b) and, thus, does not enhance the mixing in any noticeable way.

On the contrary, the two-wave solution shows strong mixing comparable to that achieved in the non-magnetic case; but at the early linear and weakly nonlinear stages, the couple of symmetric oblique waves is unable to match the effect of growing two-dimensional billows (see figure $13 \mathrm{~b}$ ). The explanation is a lower linear growth rate $\left(\beta_{\max }=0.192\right.$ at $N=0$ and $\beta_{\max }=0.0529$ at $\left.N=0.5\right)$. At later times, the 

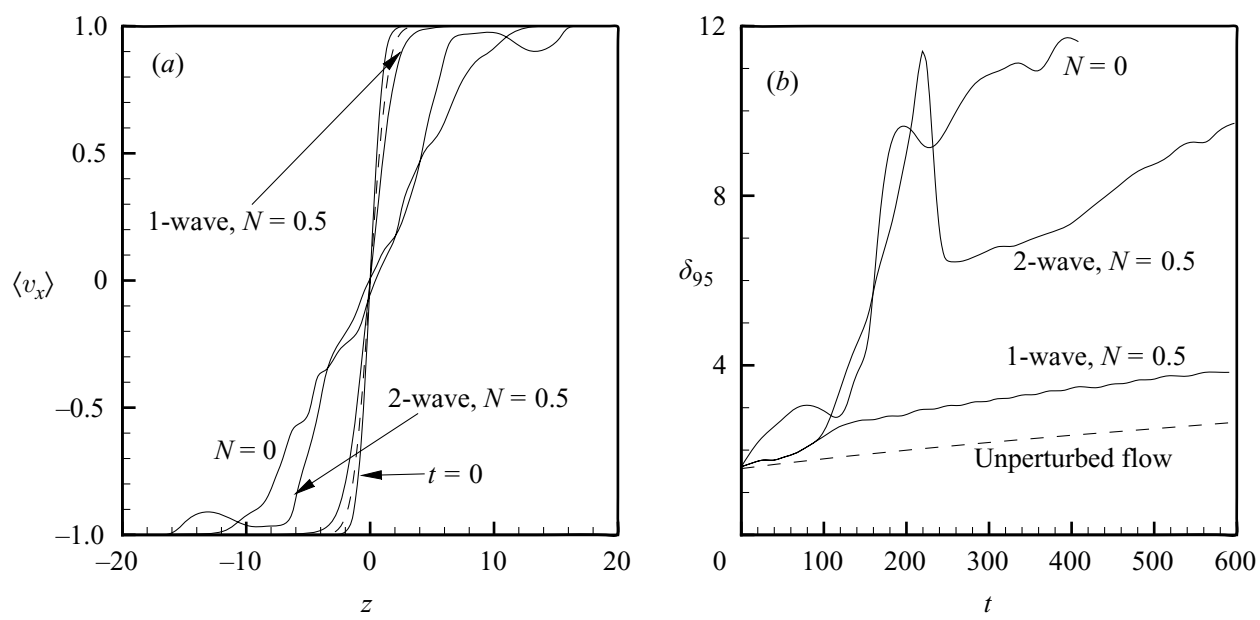

FIGURE 13. Effect of magnetic field and initial conditions on the thickness of the turbulent mixing layer. $R e=500, N=0.5$ and 0 . Initial conditions with one and two waves are used for simulations at $N=0.5$. $(a)$ - profiles of mean velocity $\left\langle v_{x}\right\rangle$ at the initial moment and the moment of developed turbulent flow $(t \approx 295)$. - - - diffusive profile. $(b)$ Thickness $\delta_{95}$ of the mixing layer as a function of time. Diffusion of the unperturbed flow (---) is shown for comparison.

inherent three-dimensionality of the two-wave solution gives rise to a fast transition to turbulence and enhancement of mixing, while the development of the non-magnetic perturbations is 'arrested' in the phase of quasi-steady quasi-two-dimensional billows. This results in the comparable thicknesses in the two-wave and non-magnetic cases during a long period of time (approximately between $t \approx 100$ and $t \approx 200$, as shown in figure $13 b$ ). At later stages, the magnetic suppression of turbulence results in some reduction of mixing. As indicated in figure 13(a), the reduction occurs primarily in the region of strong shear around the midplane. It can also be seen in figure $13(a, b)$ that the magnetic field cannot prevent excursions of strong turbulent plumes into the outer region (to distances of more than 10 from the midplane).

Finally, we examine how the strength of the applied magnetic field affects the instability and turbulence. The results of three numerical experiments performed at $R e=500$ and three values of the magnetic interaction parameter $N=0,0.5$ and 1.0 are presented in figure 14. The initial conditions in all simulations are formed as combinations (3.2) of the basic flow, the most unstable eigenmodes and the random field. The amplitudes $A_{1}$ and $A_{2}$ are selected so that they provide equal distributions of initial energy in all cases. A superposition of two symmetric oblique waves is used as an eigensolution in the cases with non-zero magnetic field.

The flow at $N=1$ demonstrates the same fundamental features as the flow at $N=0.5$ described above. Most importantly, unlike the non-magnetic flow, it does not experience a lengthy phase of growth and nonlinear saturation of the quasitwo-dimensional billows with their subsequent secondary instability. The transition to turbulence follows the linear growth in a continuous way (see the growth of $E^{\prime}$ in figure $14 b$ ).

Comparing the solutions for $N=0.5$ and $N=1$, we observe one noticeable difference. The transition to turbulence, marked by a sharp increase of the viscous dissipation rate, occurs at a much later time at $N=1$. This can be explained by the lower linear growth $\left(\beta_{\max }=5.29 \times 10^{-2}\right.$ at $N=0.5$ and $\left.\beta_{\max }=2.65 \times 10^{-2} N=1\right)$. 
(a)

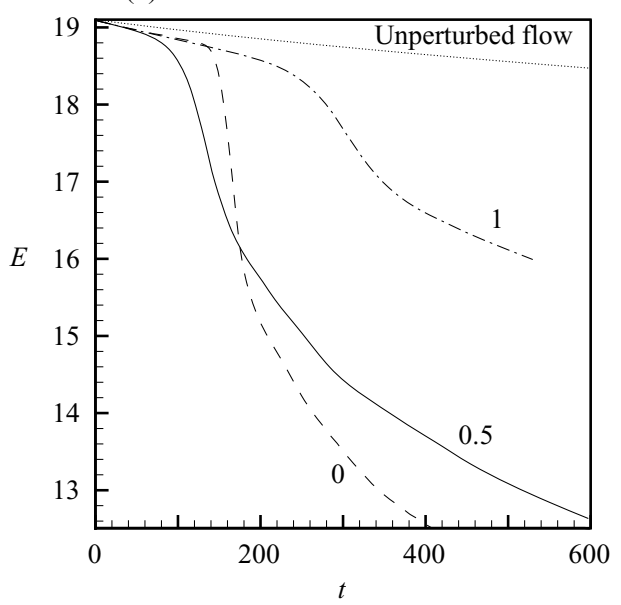

(b)

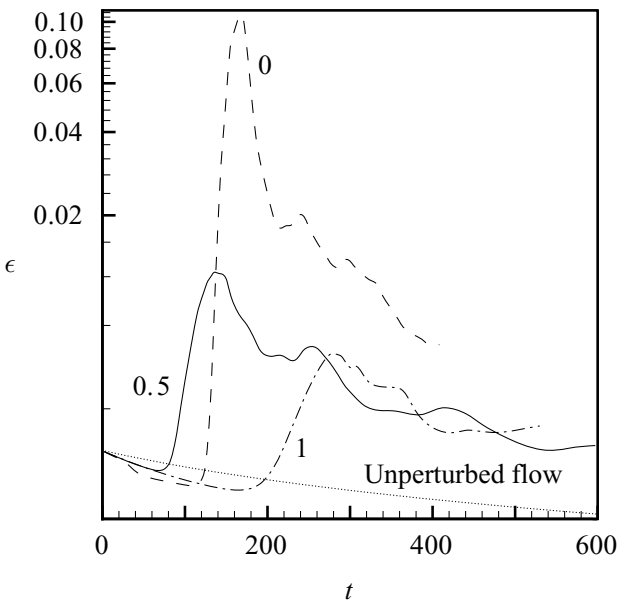

(b)

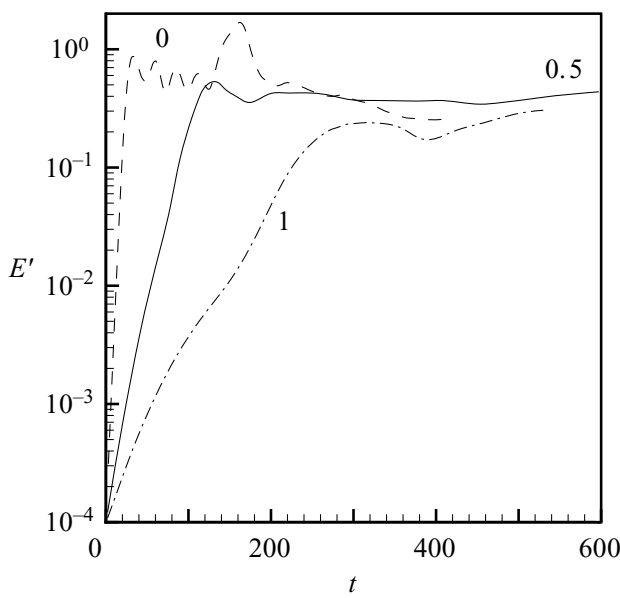

$(d)$

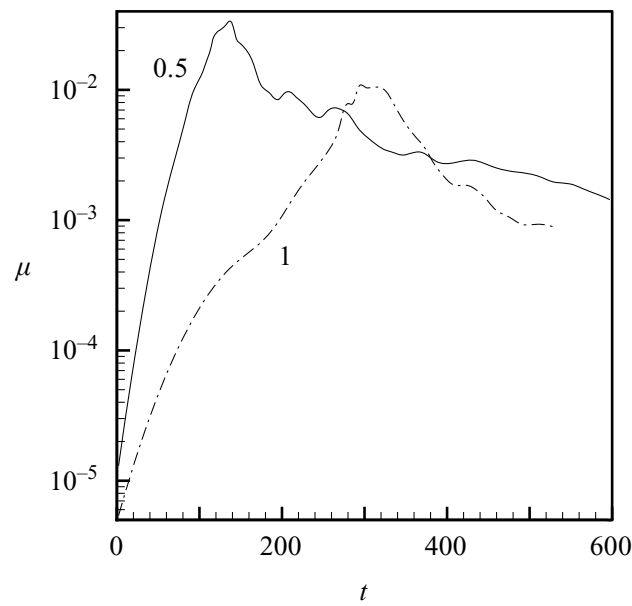

FiguRE 14. Evolution of total energy $(a)$, energy of three-dimensional perturbations of the mean flow $(b)$, viscous $(c)$ and magnetic $(d)$ dissipation rates. $---, N=0, k_{\max }=0.43, \theta_{\max }=0$; $-, N=0.5, k_{\max }=0.323, \theta_{\max }=0.262 \pi ;-\cdot-, N=1, k_{\max }=0.43, \theta_{\max }=0.39 \pi$. $\cdots$, diffusion of the unperturbed basic flow. All simulations are for $R e=500$. The transition at $N=0.5$ and $N=1$ is initiated by a combination of two oblique waves.

There is also some reduction in the strength of turbulence as demonstrated by the lower peak values of $E^{\prime}, \epsilon$ and $\mu$. In view of the delayed transition at $N=1$, it is unclear whether the main reason for the reduction is the direct suppression of the fluctuations by the magnetic field or the diffusion of the mean velocity profile over a longer period of time.

\section{Concluding remarks}

In this paper, we revisited the instability of a free shear layer (modelled as a temporally evolving flow initially given by the erf-function velocity profile) subject to a parallel uniform magnetic field. The case of small magnetic Reynolds number was considered. We provided detailed analysis of the linear instability and illustrated 
the evolving nonlinear regimes and transition to turbulence in direct numerical simulations.

Our analysis confirms the earlier predictions of Hunt (1966) and Gotoh (1971) that the instability occurs because of three-dimensional disturbances if the applied magnetic field is sufficiently strong $(N>0.106)$. The three-dimensional eigenmodes have the form of horizontal rolls, which are not perpendicular to the direction of the basic flow. The oblique angle $\theta$ measured as an angle between the wavenumber vector of the perturbation and the flow direction increases with the strength of the magnetic field and tends to $\pi / 2$ as $N \rightarrow \infty$.

We investigated the fastest growing perturbations at supercritical Reynolds numbers. At $N$ between 0.106 and 0.339 , such perturbations are three-dimensional only in a finite range of Reynolds numbers, becoming two-dimensional at higher $R e$. At $N>0.339$, the fastest growing perturbations are always three-dimensional and have the form of oblique rolls. The strength of the magnetic field affects only the spatial orientation of these modes. The oblique angle increases with $N$, while the horizontal and vertical dimensions, and the spatial shape of the rolls remain unchanged.

We performed direct numerical simulations of the transition to turbulence resulting from the instability. Two types of initial conditions were considered. In both cases, the initial velocity field consisted of the basic flow, the fastest growing eigenmode and low-amplitude noise. The difference was in the composition of the eigenmodes, for which a single oblique wave (one-wave solution) or a combination of two symmetric oblique waves with positive and negative $\theta$ (two-wave solution) were taken. We have found that the flow evolution strongly depends on the form of the initial disturbance. Two symmetric waves result in stronger nonlinear growth, excitation of a wider range of length scales, stronger vorticity, and, generally, faster transition to more intense turbulence. This is accompanied by much stronger turbulent mixing, which, at $N=0.5$, is comparable with the mixing in the non-magnetic case at the same $R e$.

We analysed the evolution of the two-wave solution and found it to be principally different from the well-studied scenarios of the classical non-magnetic KelvinHelmholtz instability. The first stage of the Kelvin-Helmholtz transition is always the development of two-dimensional billows. The three-dimensionality and turbulence appear as results of the secondary instability through pairing, spanwise waves in the braid region, etc. On the contrary, the two growing symmetric oblique waves generate the three-dimensionality immediately, in their direct interaction with each other, basic flow and the magnetic field. We have seen that this interaction can lead to a fast transition to turbulence and efficient mixing.

Another important difference between the MHD and the non-magnetic cases is the state of the developed turbulent mixing layer. Not only does the magnetic field add suppression by the Joule dissipation, it also introduces anisotropy. The flow structures are elongated in the direction of the magnetic field. We saw that, as is typical for the decaying turbulence, the anisotropy is quite strong even at moderate magnetic interaction parameters such as $N=0.5$.

In real flows, the unstable perturbations occur in a wide range of horizontal wavenumbers $k_{x}$ and $k_{y}$ with positive and negative oblique angles. The transition is likely to involve the interaction between several such modes in a manner consistent with our two-wave solution.

What would be the strength of the magnetic field, at which the phenomena discussed in the paper would appear in laboratory or industrial flows? We can make a simple estimate by considering a 'typical' liquid metal of density $\rho=10^{4} \mathrm{~kg} \mathrm{~m}^{-3}$ and electric 
conductivity of $\sigma=10^{6} \mathrm{~S} \mathrm{~m}^{-1}$. For a flow with $L=0.1 \mathrm{~m}$ and $U=1 \mathrm{~m} \mathrm{~s}^{-1}$, the critical magnetic field corresponding to $N_{F} \approx 0.1$, i.e. the field above which the first instability is to an oblique wave, is between 0.03 and $0.1 \mathrm{~T}$. Such values are commonly exceeded in experiments and in industrial processes.

Our last comment suggested by a reviewer concerns the instability and transition in shear layers with other orientations of the magnetic field. A few qualitative predictions can be made without detailed analysis. A normal field $\boldsymbol{B}=(0,0, B)$ would interact with the basic flow (1.1) and generate the Lorentz force (in non-dimensional form) $N(\boldsymbol{j} \times \boldsymbol{B})=(-N U(z), 0,0)$, i.e. the force tending to eliminate the velocity gradient in the $z$-direction and to accelerate the decay. The normal field is not expected to modify the symmetry of the unstable waves as the parallel field does in our paper, but it can modify the spatial shape of the spanwise and three-dimensional perturbations through direct suppression and accelerated decay of the basic shear.

A spanwise field $\boldsymbol{B}=(0, B, 0)$ does not interact with the basic flow or with purely spanwise perturbations. The first stage of the classical hydrodynamic instability, namely growth and saturation of two-dimensional billows would, therefore, not be affected. The subsequent three-dimensional breakdown and transition to turbulence would, however, be delayed and suppressed. This opens the possibility of finiteamplitude spanwise billows stabilized by the magnetic field and evolving, at sufficiently high Reynolds numbers, into 'quasi-two-dimensional' turbulence. The term 'quasi' is used here as a precaution since it is still unclear whether 'true' two-dimensional turbulence can be realized in three-dimensional systems, even in the presence of a very strong magnetic field. Discussions of this can be found, for example, in Tsinober (1990) and Thess \& Zikanov (2005).

The work was started during the authors' participation in the 2005 MHD Summer Program at the Université Libre de Bruxelles, Belgium. The authors wish to thank the organizers, especially D. Carati and B. Knaepen, for their hospitality. The work was supported by grant DE FG02 03 ER46062 from the US Department of Energy. The authors have benefited from fruitful discussions with A. Thess, J.C. R. Hunt, K. Moffatt, and S. Molokov. Special acknowledgment is to P. Sarathy for his help in developing the parallel code for direct numerical simulations.

\section{REFERENCES}

Abas, S. 1969 The effect of a parallel magnetic field on the stability of free boundary-layer type flows of low magnetic Reynolds number. J. Fluid Mech. 38, 243-253.

Betchov, R. \& SzewczYK, A. 1963 Stability of a shear layer between parallel streams. Phys. Fluids 6(10), 1391.

Davidson, P. A. 2001 An Introduction to Magnetohydrodynamics. Cambridge University Press.

DrazIN, P. G. 1960 Stability of parallel flow in a parallel magetic field at small magnetic Reynolds numbers. J. Fluid Mech. 8, 130.

Drazin, P. G. \& ReID, W. H. 1981 Hydrodynamic Stability. Cambridge University Press.

Gотон, K. 1971 Hydromagnetic instability of a free shear layer at small magnetic Reynolds numbers. J. Fluid Mech. 49, 21.

Hunt, J. C. R. 1966 On the stability of parallel flows with parallel magnetic fields. Proc. R. Soc. Lond. A 293, 342.

Kerswell, R. R. 2002 Elliptical instability. Annu. Rev. Fluid Mech. 34, 83-113.

Maslowe, S. A. 1981 Shear Flows Instabilities and Transition. In Hydrodynamic Instabilities and the Transition to Turbulence, (ed. H. L. Swinney \& J. P. Gollub), p. 181.

Metcalfe, R. W., Orszag, S. A., Brachet, M. E., Menon, S. \& Riley, J. J. 1987 Secondary instability of a temporally growing mixing layer. J. Fluid Mech. 184, 207-243. 
Michael, D. H. 1953 The stability of plane parallel flows of electrically conducting fluids. Proc. Camb. Phil. Soc. 49, 166.

MoreaU, R. 1990 Magnetohydrodynamics, Kluwer.

MÜLleR, U. \& BüHLER, L. 2001 Magnetohydrodynamics in Channels and Containers. Springer.

Rogers, M. M. \& Moser, R. D. 1992 The three-dimensional evolution of a plane mixing layer: the Kelvin-Helmholtz rollup. J. Fluid Mech. 243, 183-226.

Rogers, M. M. \& Moser, R. D. 1993 Spanwise scale selection in plane mixing layers. J. Fluid Mech. 247, 321-337.

Squire, H. B. 1933 On the stability of three-dimensional disturbances of viscous fluid flow between parallel walls Proc. R. Soc. Lond. A 142, 621.

StUART, J. T. 1954 On the stability of viscous flow between parallel planes in the presence of a coplanar magnetic field. Proc. R. Soc. Lond. A 221, 189.

Thess, A. \& ZiKanov, O. 2005 On the transition from two-dimensional to three-dimensional MHD turbulence. Proc. of 2004 CTR Summer Program, Stanford University, pp. 63-74.

TsinOBER, A. 1990 MHD flow drag reduction. In Viscous Drag Reduction in Boundary Layers. (ed. D. M. Bushnell \& J. N. Hefner), pp. 327-349.

Vorobev, A., Zikanov, O., Davidson, P. \& KnaEPEN, B. 2005 Anisotropy of MHD turbulence of low magnetic Reynolds number. Phys. Fluids 17(12), 125105.

Zikanov, O. \& Thess, A. 1998 Direct numerical simulation of forced MHD turbulence at low magnetic Reynolds number. J. Fluid Mech. 358, 299-333.

Zikanov, O., Slinn, D. N., \& DhanaK, M. R. 2004 Turbulent convection driven by surface cooling in shallow water $J$. Fluid Mech. 464, 81-111. 